OPEN ACCESS

Edited by:

Chengdao $L i$,

Murdoch University, Australia

Reviewed by:

Yan Long,

Chinese Academy of Agricultural

Sciences, China

Liezhao Liu,

Southwest University, China

*Correspondence:

Santosh K. Upadhyay

skupadhyay@pu.ac.in;

santoshpandit16@gmail.com

Specialty section: This article was submitted to Crop Science and Horticulture,

a section of the journal

Frontiers in Plant Science

Received: 26 May 2016

Accepted: 29 August 2016

Published: 22 September 2016

Citation:

Shumayla, Sharma S, Kumar R, Mendu V, Singh K and Upadhyay SK

(2016) Genomic Dissection and

Expression Profiling Revealed

Functional Divergence in Triticum

aestivum Leucine Rich Repeat

Receptor Like Kinases (TaLRRKS).

Front. Plant Sci. 7:1374.

doi: 10.3389/fp/s.2016.01374

\section{Genomic Dissection and Expression Profiling Revealed Functional Divergence in Triticum aestivum Leucine Rich Repeat Receptor Like Kinases (TaLRRKs)}

\author{
Shumayla ${ }^{1,2}$, Shailesh Sharma ${ }^{3}$, Rohit Kumar ${ }^{2}$, Venugopal Mendu ${ }^{4}, K^{2}$ ashmir Singh ${ }^{2}$ and \\ Santosh K. Upadhyay ${ }^{1 *}$ \\ ${ }^{1}$ Deparment of Botany, Panjab University, Chandigarh, India, ${ }^{2}$ Deparment of Biotechnology, Panjab University, Chandigarh, \\ India, ${ }^{3}$ National Agri-Food Biotechnology Institute, Mohali, India, ${ }^{4}$ Department of Plant and Soil Science, Fiber and \\ Biopolymer Research Institute, Texas Tech University, Lubbock, TX, USA
}

The leucine rich repeat receptor like kinases (LRRK) constitute the largest subfamily of receptor like kinases (RLK), which play critical roles in plant development and stress responses. Herein, we identified 531 TaLRRK genes in Triticum aestivum (bread wheat), which were distributed throughout the $A, B$, and $D$ sub-genomes and chromosomes. These were clustered into 233 homologous groups, which were mostly located on either homeologous chromosomes from various sub-genomes or in proximity on the same chromosome. A total of 255 paralogous genes were predicted which depicted the role of duplication events in expansion of this gene family. Majority of TaLRRKs consisted of trans-membrane region and localized on plasma-membrane. The TaLRRKs were further categorized into eight phylogenetic groups with numerous subgroups on the basis of sequence homology. The gene and protein structure in terms of exon/intron ratio, domains, and motifs organization were found to be variably conserved across the different phylogenetic groups/subgroups, which indicated a potential divergence and neofunctionalization during evolution. High-throughput transcriptome data and quantitative real time PCR analyses in various developmental stages, and biotic and abiotic (heat, drought, and salt) stresses provided insight into modus operandi of TaLRRKs during these conditions. Distinct expression of majority of stress responsive TaLRRKs homologous genes suggested their specified role in a particular condition. These results provided a comprehensive analysis of various characteristic features including functional divergence, which may provide the way for future functional characterization of this important gene family in bread wheat.

Keywords: duplication events, expression, neofunctionalization, phylogenetic groups, TaLRRKs, Triticum aestivum, stress 


\section{INTRODUCTION}

The leucine rich repeat receptor like kinases (LRRKs) represent the largest subfamily of receptor like kinases (RLK), which are involved in diverse functions in plants (Shiu and Bleecker, 2001; Diévart and Clark, 2003; Shiu et al., 2004). Similar to the other RLK proteins, LRRKs also consisted of an extracellular domain (ECD) to perceive signals, a trans-membrane region to comfort the protein within the membrane, and a cytoplasmic protein kinase domain to stimulate the plant immunity via autophosphorylation, which is followed by phosphorylation of a specific substrate (Walker and Zhang, 1990; Shiu and Bleecker, 2001; Gou et al., 2010). The ECD of LRRKs consist of LRR domain, constituted by tandem repeats of 20-30 amino acid (AA) residues long leucine rich structural motifs (Jones and Jones, 1997; Matsushima and Miyashita, 2012). The LRR domain is stable among all proteins built from tandemly repeated motifs because of its hydrophobic solenoid inner core, where the conserved leucine and other aliphatic residues are present. This structure also favors the protein-protein interactions (Bella et al., 2008).

The LRRKs are involved in growth, development, and survival including organogenesis, morphogenesis, hormone signaling, and abiotic and biotic stress management in plants (Diévart and Clark, 2003; Li and Tax, 2013). For instance, BARELY ANY MERISTEM (BAM) 1-3 and CLAVATA1 are involved in apical growth, ERECTA-LIKE (ERL) 1-2 in organ growth, plant architecture, and stomatal and floral development, BRI1 in vascular development, HSL in flower abscission, FEI2 in cell wall biosynthesis, and GSO and RPK1 in embryogenesis, (Clark et al., 1997; Delgado et al., 2004; Shpak et al., 2004; Deyoung et al., 2006; Nodine et al., 2007; Stenvik et al., 2008; Tsuwamoto et al., 2008; Xu et al., 2008). The LRRK FLS2 and EFR regulate the plant immunity and act as receptor for flagellin and EF-Tu for plant defense (Gomez and Boller, 2000; Zipfel et al., 2006). PSKR1, BAK1, BRI1, and SERK of arabidopsis are also reported to be involved in biotic stress and defense responses ( $\mathrm{Nam}$ and $\mathrm{Li}$, 2002; Roux et al., 2011; Wang et al., 2011; Mosher et al., 2013; Wu et al., 2016). The differential expression of LRRK genes during abiotic stresses in various plants like arabidopsis, rice, and potato has been reported (Diévart and Clark, 2004; Osakabe et al., 2005; Park et al., 2014; Wu et al., 2016). The GHR1 of arabidopsis is induced by $\mathrm{ABA}$ and $\mathrm{H} 2 \mathrm{O} 2$, while RPK1 by drought, salt, abscisic acid (ABA), and low temperature (Osakabe et al., 2005; Hua et al., 2012). OsGIRL1 is involved in salt, osmotic, heat, and gamma radiation stress in Oriza sativa (Park et al., 2014). Several other differentially expressed LRRKs are known in response to various biotic and abiotic stresses in plants, whose specific functions need to be established in future studies. Moreover, some LRRKs like SOMATIC EMBRYOGENESIS RECEPTOR-LIKE KINASE (SERK) and ERECTA of arabidopsis (Godiard et al., 2003; Shpak et al., 2004; Colcombet et al., 2005) possess dual functions, which

Abbreviations: AA, Amino acids; DS, Drought stress; F, folds; h, hour; HD, Combined heat and drought stress; HS, Heat stress; SD, segmental duplication; TaLRRK, T. aestivum Leucine rich repeat receptor like kinases; TD, Tandem duplication. are due to either recognition of numerous ligands by a single receptor or cross talk between various pathways (Torii et al., 1996; Afzal et al., 2008). The LRRKs are mostly evolved by extensive gene/domain duplication; therefore they have probably acquired diverse functions through neofunctionalization (Jones and Jones, 1997; Walsh, 2003).

In plants, the functional characterization of $L R R K$ genes are largely performed in model plant arabidopsis (Shiu and Bleecker, 2001; Shiu et al., 2004; Wu et al., 2016). In recent years, the study has been certainly extended, but it is still limited to a few plants like rice, tomato brassica, poplar, and soybean (Sun and Wang, 2011; Zan et al., 2013; Rameneni et al., 2015; Wei et al., 2015; Zhou et al., 2016). It is probably due to lack of genomics information, large number of genes and complication in applicability of functional genomics tools in various other plants. The Triticum aestivum (bread wheat) is one of the main food crops of the world, which is being consumed by about one-third population as a major source of calorie. It is used in the preparation of bread, biscuit, cake, pastries, and several other food materials. The T. aestivum comprises a composite allohexaploid genome $(2 \mathrm{n}=6 \mathrm{x}=42$; AABBDD), which was originated by hybridizations of three diploid A, B, and D sub-genomes (Marcussen et al., 2014). The characterization of LRRK gene family has not been performed in T. aestivum, despite being a staple food crop. The International Wheat Genome Sequencing Consortium (IWGSC) has reported the chromosome based genome information of T. aestivum in 2014 (IWGSC, 2014). Further, numerous developmental stage specific and stress related high-throughput RNA sequence (RNA seq) data of $T$. aestivum have been reported in recent years (Zhang et al., 2014, 2016; Liu et al., 2015; Pingault et al., 2015). The availability of genomic information and these RNA seq data enabled us for the identification and characterization of LRRK gene family in $T$. aestivum. Since the $L R R K$ genes play vital roles in development and stress tolerance in plants (Shiu et al., 2004), their characterization will be valuable in future genetic improvement program of T. aestivum.

Herein, the entire TaLRRK gene family was identified using the genomic data of T. aestivum (IWGSC, 2014) and comprehensive analyses were performed. The gene family was characterized for genome wide distribution, occurrence of duplication events, homologous, and orthologous genes identification, annotation, and gene ontology (GO) mapping, gene, and protein structure, domain, and motif organization, and evolutionary relationships. The expression profiling during various developmental stages and stress conditions was performed to understand their role in these biological processes. To the best of our knowledge, this is the first report on genome wide characterization of $L R R K$ genes in T. aestivum.

\section{MATERIALS AND METHODS}

\section{Identification, Annotation, and Chromosomal Distribution}

To identify the entire TaLRRKs of $T$. aestivum, following two approaches were used. First, we performed the blastp search of known LRRK protein sequences of arabidopsis and rice 
(Shiu and Bleecker, 2001; Shiu et al., 2004; Sun and Wang, 2011) against high confidence protein model sequences of $T$. aestivum obtained from the IWGSC (http://www.wheatgenome. org/, http://wheat-urgi.versailles.inra.fr/Seq-Repository/Genesannotations) (IWGSC, 2014). Second Hidden Markov model (HMM) scan was performed using LRR (LRR_8, PF13855; LRR_NT II, PF08263; LRR_4, PF012799; LRR_6, PF13516; LRR_9, PF14580; LRR_I, PF00560; LRR_V, PF13306), and protein kinase (PF00069) pfam profiles obtained from pfam database (ftp://ftp.sanger.ac.uk/pub/databases/Pfam) (Finn et al., 2014). The unique set of sequences having both LRR and protein kinase domain were considered as putative TaLRRKs. These sequences were further validated by blast search against NCBIconserved domain database (http://www.ncbi.nlm.nih.gov/ Structure/bwrpsb/bwrpsb.cgi). Further, the domain organization was confirmed using SMART (http://smart.embl-heidelberg. de/) and Prosite-Scan (http://prosite.expasy.org/scanprosite/) servers. The annotation of identified sequences was performed by blast search $\left(e\right.$-value $\left.10^{-6}\right)$ against the NCBI non-redundant protein, UNIPROT/SWISSPROT, and UNIPROT-UNIREF databases. The BLAST2GO tool was used for the gene ontology mapping (Conesa and Gotz, 2008). The chromosomal location was obtained by blastn search of TaLRRK gene sequences against chromosome sequences of $T$. aestivum available at Ensembl Plants (http://plants.ensembl.org/Triticum_aestivum/Info/ Index) and URGI (http://urgi.versailles.inra.fr/blast/) servers. The sub-genomic (A, B, and D) categorization was based on gene model sequence identifier. MapInspect (http://mapinspect. software.informer.com/) was used to prepare the chromosome maps.

\section{Prediction of Homologs, Orthologs, Duplication Events, and Phylogenetic Analysis}

The homologous sequences were predicted on the basis of two criteria- (i) bi-directional blastn search $\left(e\right.$-value $\left.10^{-10}\right)$ with $\geq 90 \%$ sequence similarity between TaLRRK gene sequences and (ii) clustering with the same $T$. aestivum unigene sequence (http://www.ncbi.nlm.nih.gov/UniGene). The sequences following both the criteria were considered as homologs. A non-redundant nomenclature was also performed as per the clustering and homology between sequences. The orthologous genes were predicted using best bidirectional blast hit (e-value $10^{-10}$ ) approach. The TaLRRK gene sequences were used for blastn search against the $L R R K$ sequences of rice and arabidopsis. The intra-chromosomal duplication events were predicted by blastn search ( $e$-value $10^{-10}$ ) with $\geq 80 \%$ sequence similarity. The genes located within $5 \mathrm{Mb}$ region on the same pseudo-molecule (chromosome) were considered as tandemly duplicated (TD), while those located beyond $5 \mathrm{Mb}$ were designated as segmentally duplicated (SD) (Shumayla et al., 2016). The evolutionary relationship was analyzed by neighbor joining method with 1000 bootstrap replicates using MEGA7 (Kumar et al., 2016).

\section{In-silico Characterization}

The exon/intron configuration was obtained by aligning coding sequences (CDS) with genomic sequence of each gene obtained from Ensemble plants (http://plants.ensembl.org/Triticum_
aestivum/Info/Index). The conserved domains were predicted by blast search at NCBI conserved domain database (http://www. ncbi.nlm.nih.gov/Structure/bwrpsb/bwrpsb.cgi) (Marchler et al., 2015). Motifs were predicted by multiple sequence alignment subjected to the Web Logo (http://weblogo.berkeley.edu/logo. cgi) and consurf blast (http://consurf.tau.ac.il) server. The molecular weight, pI, signal peptide, cellular localization, and transmembrane region were predicted using Expasy MW/pI (http://web.expasy.org/compute_pi/), SignalP 4.1 (http://www. cbs.dtu.dk/services/SignalP/), CELLO v.2.5 (http://cello.life.nctu. edu.tw/), and TMHMM v2.0 (http://www.cbs.dtu.dk/services/ TMHMM/) servers, respectively (Yu et al., 2004, 2006). MAFFT, (http://www.ebi.ac.uk/Tools/msa/mafft/), muscle (Edgar, 2004) and ClustalW (http://www.ebi.ac.uk/Tools/msa/clustalw2/) were used for the multiple sequence alignments. The venn diagram was made using Venny (Oliveros, 2007-2015).

\section{Genome Wide Expression Analysis}

The tissue specific expression profiling of TaLRRKs was performed using high throughput RNA-seq data (https://urgi.versailles.inra.fr/files/RNASeqWheat/) generated in two biological replicates from root, stem, leaf, spike, and grain, each with three developmental stages (accession number ERP004714) (Pingault et al., 2015). The effect of biotic stress of fungus Blumeria graminis $f$. sp. tritici (Bgt) and Puccinia striiformis f. sp. tritici (Pst) on the expression of TaLRRKs in leaves was studied using RNA seq data (accession number PRJNA243835) developed after $24 \mathrm{~h}$ of inoculation in triplicates (Zhang et al., 2014). Expression analysis under heat, drought and their combination was performed using RNA seq data (accession number SRP045409) generated from leaves after 1 and $6 \mathrm{~h}$ of incubation in two biological replicates (Liu et al., 2015). The effect of salt $(\mathrm{NaCl})$ stress was analyzed in root tissue using RNA seq data (accession number SRP062745) produced in three biological replicates after $6,12,24$, and $48 \mathrm{~h}$ of treatments (Zhang et al., 2016). The quality filtered RNA seq reads were mapped to TaLRRK gene sequences with $100 \%$ query coverage and sequence similarity. The abundance of mapped reads to each gene was counted using locally made python script and used to calculate RPKM (reads per kilobase per million mapped reads) value as described (Mortazavi et al., 2008; Upadhyay et al., 2015; Shumayla et al., 2016). The heat-maps showing expression profiles were generated using Hierarchical Clustering Explorer 3.5 (http://www.cs.umd.edu/hcil/hce/).

\section{Validation of Gene Expression by qRT-PCR}

The organ specific expression of selected TaLRRK genes were validated in root, shoot, leaf, and seeds (from two developmental stages, Z_71 and Z_75; Z denotes Zadoks scale) of T. aestivum cv. Chinese Spring following the method described (Shumayla et al., 2016). The heat, drought, and their combination stress responsive expression was validated in leaves subjected to 1 and $6 \mathrm{~h}$ of incubation, while the effect of salt $(\mathrm{NaCl})$ stress on selected TaLRRK genes expression in root tissue was analyzed after 6,12 , 24, and $48 \mathrm{~h}$ of treatments, as described (Liu et al., 2015; Zhang et al., 2016). The seeds were surface sterilized using $1 \%$ sodium hypochlorite solution, washed several times using autoclaved Milli-Q water and placed on moistened filter paper in petri 
dishes for 2 days at $20^{\circ} \mathrm{C}$. The uniformly grown seedlings were transferred to half strength Hoagland solution in hydroponic condition and grown in growth chamber under $22 / 18^{\circ} \mathrm{C}$ (day/night), 16/8 h (light/dark), and 50\% relative humidity. The seedlings were subjected to various stress conditions after 1 week. The drought stress was applied using 20\% PEG solution in place of water (Dhanda et al., 2004; Soltani et al., 2006), while heat stress was applied by incubation of plants at $40^{\circ} \mathrm{C}$ in growth chamber. The leaves samples were collected separately after 1 and $6 \mathrm{~h}$ of incubation under heat $\left(40^{\circ} \mathrm{C}\right)$, drought $(20 \%$ PEG), and combination $\left(40^{\circ} \mathrm{C}\right.$ and $20 \%$ PEG) stress, frozen in liquid nitrogen, and stored at $-80^{\circ} \mathrm{C}$. The salt stress was applied by adding $150 \mathrm{mM} \mathrm{NaCl}$ to the hydroponic solution and root samples were collected after different hours of treatment. The normal grown seedlings were used as control and all the experiments were performed in three biological replicates.

The total RNA was isolated from normal and stress treated samples using the RNeasy Plant Mini Kit (Qiagen, USA) and DNA contaminations were removed using RNase free kit (Ambion USA). Superscript III First Strand cDNA Synthesis Kit (Invitrogen, USA) was used for the synthesis of cDNA and quantitative real time (RT) PCR was performed with gene specific primers (Table S1) using ABI7500 Fast System (Applied Biosystems, USA) following the method described in our earlier studies (Upadhyay et al., 2013, 2016; Shumayla et al., 2016). The amplification of 18S_rRNA gene (accession number AJ272181.1) was used as an internal control (Bhati et al., 2014; Shumayla et al., 2016) and transcripts abundance was calculated using ${ }^{\boldsymbol{\Delta}} \mathrm{Ct}$ method (Livak and Schmittgen, 2001).

\section{RESULTS}

\section{Isolation and Chromosomal Localization of TaLRRK Genes}

The blast search of LRRK protein sequences of arabidopsis and rice, HMM blast and pfam blast was used to comprehensively identify the LRRK sequences from T. aestivum. A total of 531 non-redundant TaLRRK genes were identified after an extensive blast search. The presence of an intracellular kinase (pfam00069) domain and an extracellular LRR domain in each TaLRRKs was confirmed by blast search against pfam and NCBI-conserved domain databases. These were further confirmed by PROSITEScan and SMART server.

The allohexaploid genome $(2 \mathrm{n}=6 \mathrm{x}=42$; AABBDD $)$ of $T$. aestivum was originated by hybridization events of three diploid A, B, and D sub-genomes (Marcussen et al., 2014). Genome wide distribution indicated the sharing of 166, 195, and 170 TaLRRK genes from A, B, and D sub-genomes, respectively (Figure 1A; File S1). Since the T. urartu and Aegilops tauschii are reported as donor of A and D sub-genome, (Marcussen et al., 2014) and their genome sequences are recently reported (Jia et al., 2013; Ling et al., 2013), the number of LRRK genes in these plants was also analyzed. A total of 217 and $248 L R R K$ genes were predicted in T. urartu and Ae. tauschii, respectively (File S2).

The chromosomal distribution on the basis of gene model identifier indicated the occurrence of TaLRRK genes on each chromosome but at different frequencies (Figure 1B; File S1). A maximum of 51 genes were located on chromosome 2B, while a minimum 13 genes on chromosome $5 \mathrm{~A}$. The physical localization of TaLRRK genes was analyzed by blastn search against each chromosome sequence. Out of 531 TaLRRK genes, 356 were located on pseudo-molecules (chromosomes), however remaining 175 were mapped on scaffolds (Figure 1C; File S3). This might be due to the unavailability of complete genome and chromosome sequence of T. aestivum.

\section{Prediction of Homologs, Paralogs, and Orthologs}

On the basis of high similarity with each other and, grouping with a common unigene cluster, the TaLRRK genes were clustered into 233 distinct groups with one or more sequences. These clustered sequences were considered as putative homologs on the basis of

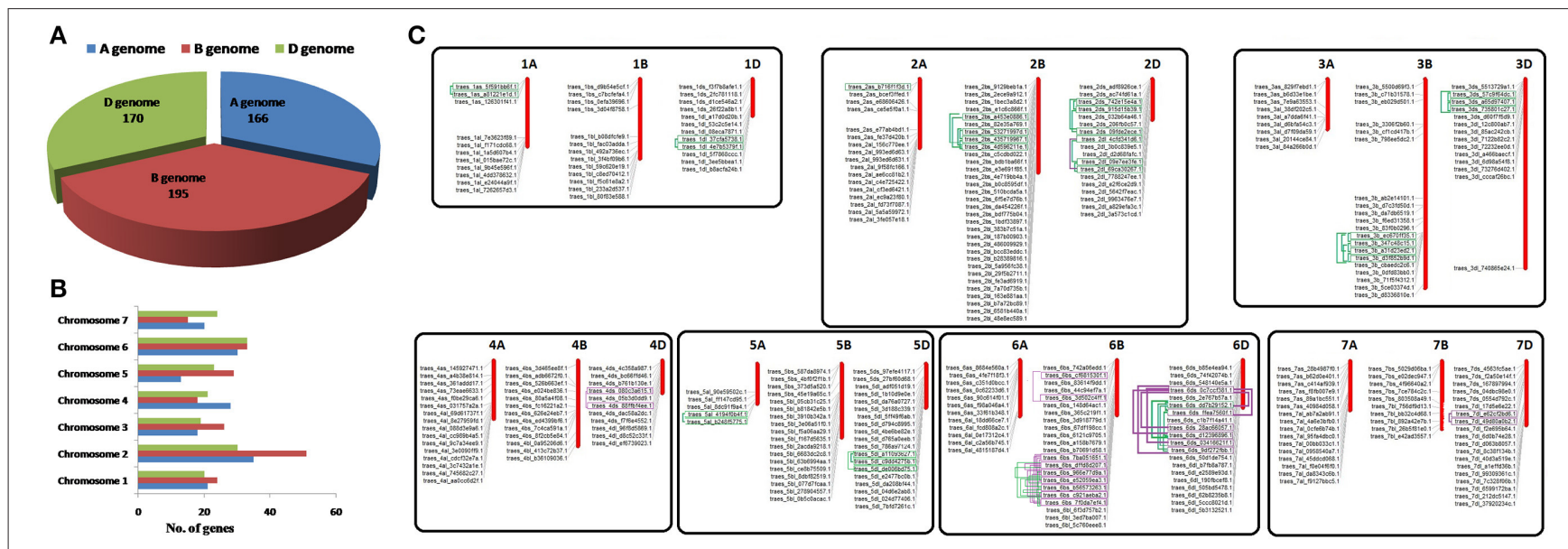

FIGURE 1 | Distribution of TaLRRK genes on A,B,D sub-genomes and chromosomes, and duplication events. Figure shows the number of TaLRRK genes located on A, B, and D sub genomes (A) and various chromosomes (B). The physical localization of TaLRRK genes on various chromosomes (C) is performed for the genes located on reported pseudo-molecules (chromosome) sequences, whereas those occurred on scaffolds are not mapped. The predicted intra-chromosomal duplication events are highlighted and connected by boxes and lines. The purple and green colors indicate segmental (SD) and tandem duplication (TD), respectively. 
their high similarity with each other. The clustering information was also used for the nomenclature of these genes (File S3). Most of the clusters consisted of at least one sequence from the homeologous chromosomes of A, B, and D sub-genomes, which might also be considered as homeologous genes. The largest cluster (LRRK70) consisted of 20 homologous sequences, while 99 TaLRRK sequences were distinct without any homolog.

A total of 255 duplication events (DEs) were predicted in TaLRRKs, out of them only 66 could classified as TD and $\mathrm{SD}$, due to the physical localization of paralogous gene pairs on pseudo-molecules (Figure 1C; File S4). However, 189 DEs could not be categorized as TD or SD, because one or both the genes from paralogous pair were located on scaffold. A total of 50,128, and 77 DEs were predicted on A, B and D sub-genomes, respectively. These DEs were localized on all the chromosomes except chromosome 7A. A maximum of 54 DEs occurred on chromosome $1 \mathrm{~B}$, while only one $\mathrm{DE}$ was located on chromosome 4B.

The reported $L R R K$ gene sequences of rice and arabidopsis was downloaded from Rice Genome Annotation Project (rice.plantbiology.msu.edu/) and The Arabidopsis Information Resource (TAIR) (www.arabidopsis.org) databases and used for the prediction orthologous sequences of TaLRRK genes by the best bidirectional blast hit approach as described earlier (Herve et al., 1999; Shumayla et al., 2016). A total of 104 and 176 nearest orthologous genes of TaLRRKs were predicted from arabidopsis and rice, respectively (File S5). The functional correlation of orthologous gene pairs has been discussed in later section.

\section{Annotation and Gene Ontology Analysis}

The identified TaLRRK genes were further validated by their functional annotation and gene ontology (GO) mapping. Annotation was performed by blast search against various databases (Files S6, S7). About 98\% TaLRRKs were annotated with $\geq 60 \%$ similarity against NCBI non-redundant protein database (Figure 2A). The top blast hit plant species were Ae. tauschii (339) and Hordeum vulgare (99) (Figure 2B). The blast search of TaLRRKs showed homology with 336 and 89 distinct protein sequences from UNIREF_100 and UNIPROT databases, respectively (File S7). Most of the TaLRRKs were annotated as leucine rich repeat receptor like serine threonine protein kinase. Some of them were annotated as homologs of arabidopsis BRASSINOSTEROID INSENSITIVE 1 (BRI1), CLAVATA1, ERECTA LIKE 1 (ERL1), ERL2, RECEPTOR LIKE PROTEIN KINASE2 (RPK2), GASSHO1 (GSO1), TMK1, MRH1, PHYTOSULFOKINE RECEPTOR 1-LIKE (PSKR1), SOMATIC EMBRYOGENESIS RECEPTOR KINASE (SERK1), and other proteins.

The GO mapping was performed using BLAST2GO tool (Conesa and Gotz, 2008). A total of 6709 GO terms were mapped to TaLRRK proteins, which were classified into cellular components (2662), biological processes (2687), and molecular functions (1360) categories. The GO term "membrane" was obtained for almost each TaLRRK in cellular component category (Figure 2C). In molecular function, binding (38\%), and catalytic activity (38\%) was enriched category (Figure 2D). In biological processes, GO terms were evenly distributed in various categories like signaling, response to stimulus, cellular, and metabolic processes (Figure 2E), which depicted diverse role of TaLRRKs in plant development and defense mechanism. Similar GO categorization is also reported for brassica LRRKs (Rameneni et al., 2015).

\section{Gene and Protein Characterization}

The gene structure was analyzed in terms of exon/intron organization. The number of exons in TaLRRK genes varied from one to 28 (File S3). Out of 531 TaLRRK genes, 71 (13\%) were intron less, and 198 (37\%) consisted of single intron (Figure 3A). A maximum of 27 introns were detected in LRRK69.1, followed by $L R R K 136.1$, and $L R R K 193.2$ with 26 introns each.

The LRRK186.1 (1315 AA) and LRRK 51.2 (169 AA) were the longest and the smallest TaLRRK proteins. However, the average coding sequence and protein length was $2386 \mathrm{bp}$ and 794 AA residues, respectively. The average molecular weight of TaLRRKs was found to be $\sim 80 \mathrm{kDa}$. The isoelectric point range of TaLRRKs was 4.8-9.9; where LRRK227 had the highest pI (File S3). Out of 531 TaLRRKs, 510 were consisted of one or more transmembrane regions. A maximum of three transmembrane regions were found in six TaLRRKs, while single transmembrane was detected in 401 TaLRRK proteins. Analysis at SignalP (http://www.cbs.dtu.dk/services/SignalP/) server indicated the presence of signal peptide in 244 TaLRRK proteins. Most of the TaLRRK proteins were predicted to be localized in plasma membrane (324), which was followed by extracellular (93), and nuclear (65) localization. A few were localized in chroloplast (29), cytoplasm (20), and mitochondria (5) (File S3).

To explore the diversification and functional potentials of TaLRRKs, their domains and motifs were analyzed. In total, 12 domains were predicted including kinase domain, which were present in various combinations (File S8). The LRR_8 and kinase domains were found in each TaLRRK proteins, which were followed by LRR_4 (494) and LRR_NT II (258). Besides these major domains, some TaLRRK also consisted of LRR_6 (34), LRR_1 (22), LRR_5 (15), LRR_9 (6), Malectin (12), P-loop NTPase (1), Aldolase II (1), and Herpes_gE (1) domains. The majority $(\sim 42 \%)$ of TaLRRKs consisted of the combination of LRR_8; LRR_NT II; LRR_4 domains, followed by LRR_8; LRR_4 domains ( $36 \%)$ along with the kinase domain (Figure 3B).

The TaLRRKs were further analyzed for conserved nature of major domains and various other motifs by multiple sequence alignment subjected to the Web Logo software and consurf blast. The kinase domain was found highly conserved in comparison to the LRR domain (Figure 3C). Several conserved motifs were detected in both LRR and Kinase domains of TaLRRK (Figure 3C; Table S2). Numerous LRR motifs were found in TaLRRKs, most of them consisted of similar organization of conserved amino acids (GxIPxxLxxLxxLxxLxLxxN). The highly conserved amino acids positions were $\mathrm{G}$ at 1 st, $\mathrm{P}$ at 4 th, $\mathrm{L}$ at 7 th, 10th, 13th, 16th, and 18th, and $\mathrm{N}$ at 21st. A few conserved motifs were also predicted in kinase domain, which were localized around the ATP binding sites, active sites, substrate binding sites, and activation loop (Figure 3C). 
A

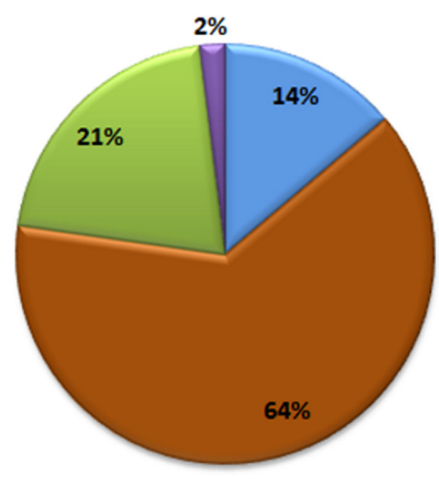

B

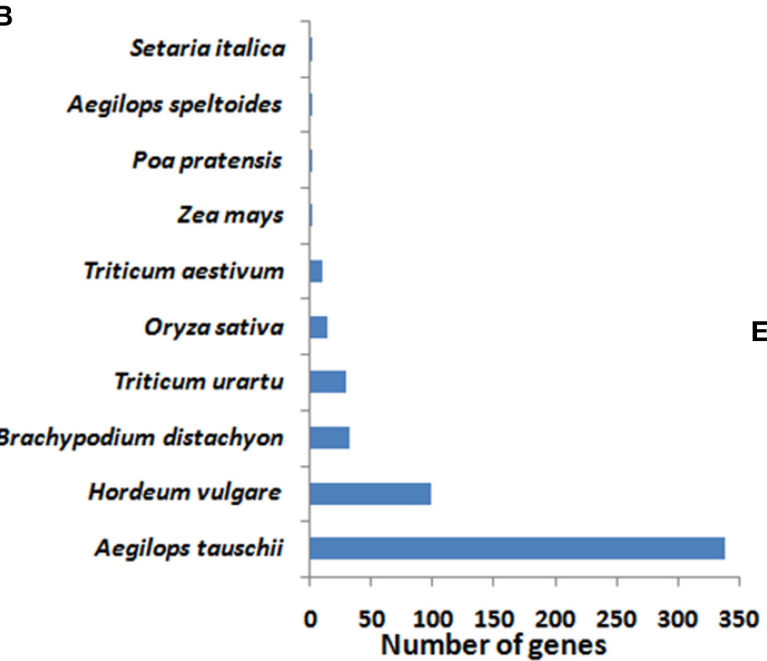

C

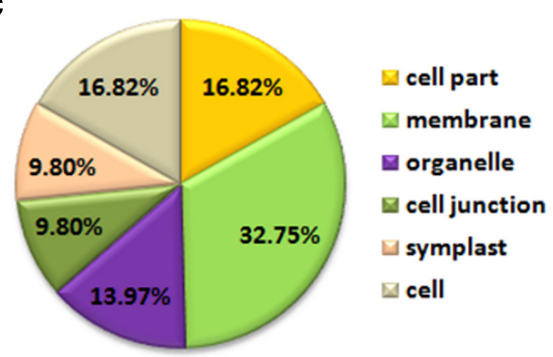

D

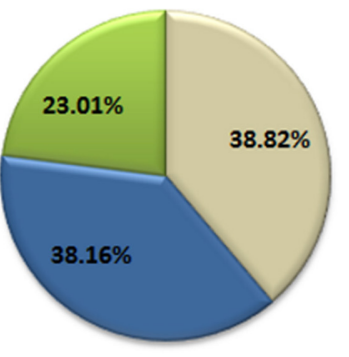

E

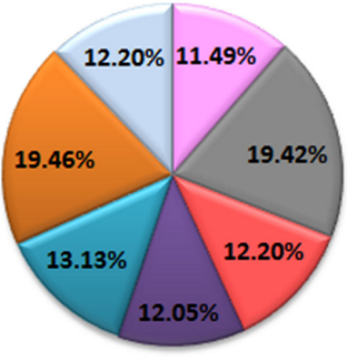

$\square$ catalytic activity

binding

$\square$ molecular

transducer activity

signaling

cellular process

$\square$ regulation of biological

process

$\square$ response to stimulus

$\square$ single-organism process

$\square$ metabolic process

$\triangle$ biological regulation

FIGURE 2 | Blast2Go annotation statistics and gene ontology (GO) mapping of TaLRRK genes. Figure shows (A) percent similarity of TaLRRK genes with the known sequences in the NCBI-nr database during annotation, and (B) top blast hit species. GO mapping shows functional categorization of TaLRRKs into (C) cellular components, (D) molecular functions and (E) biological processes.

\section{Phylogenetic Analysis}

The evolutionary relationship between 531 TaLRRK proteins was studied by constructing a phylogenetic tree using MEGA7 (Kumar et al., 2016). The TaLRRKs were classified into eight main groups, which were further divided into subgroups and clades (Figure 4). The size of each group varied significantly from two members in group I to 157 in group 7. The predicted homologous TaLRRK sequences were grouped together with high bootstrap value in phylogenetic tree, which also supported the proposed nomenclature pattern. Majority of subgroups or clades comprised either predicted homologous sequences located on homeologous chromosome (A, B, and/or D subgenome), or predicted paralogous sequences probably originated by duplication events and located on same chromosome. For instance, LRRK12 with 17 homologs was clustered in a clade of group III, while LRRK70 with 20 homologs was grouped together in group $\mathrm{V}$.

The phylogenetic groups were also analyzed in terms of exon/intron pattern (Figure 3A) and various other features (Table S3). Though the range of number of introns in each group was overlapping, the closely clustered TaLRRKs in various subgroups and clades showed a similar exon/intron organization pattern. The analysis of various other characteristic features indicated variation in average molecular weight, $\mathrm{pI}$ and signal peptide between different groups, but the range was overlapping (Table S3). The domain architecture and LRR repeats were also found consistent in closely clustered sequences in each group (File S9).

\section{Expression Analysis in Various Organs and Developmental Stages}

The expression profiling of TaLRRK genes in various organs and their developmental stages was performed using high throughput RNA-seq data (Pingault et al., 2015). A total of 263 genes were used for expression analysis, because their expression values were $\geq 10$ RPKM in one or more developmental stages (File S10). The organ wise expression profiling indicated that majority of TaLRRK genes were highly expressed in root $(\sim 40)$ and spike $(\sim 35 \%)$ compared to the other organs (Figure 5A). Only six genes (three homologs of LRRK79, two of LRRK40, and LRRK178.1) showed higher expression in grain than in other organs. The real time (RT) PCR validation of certain genes like showed a similar relative expression pattern (Figure $5 \mathrm{H}$ ). For instance, the genes LRRK7, LRRK177, and LRRK180 were 


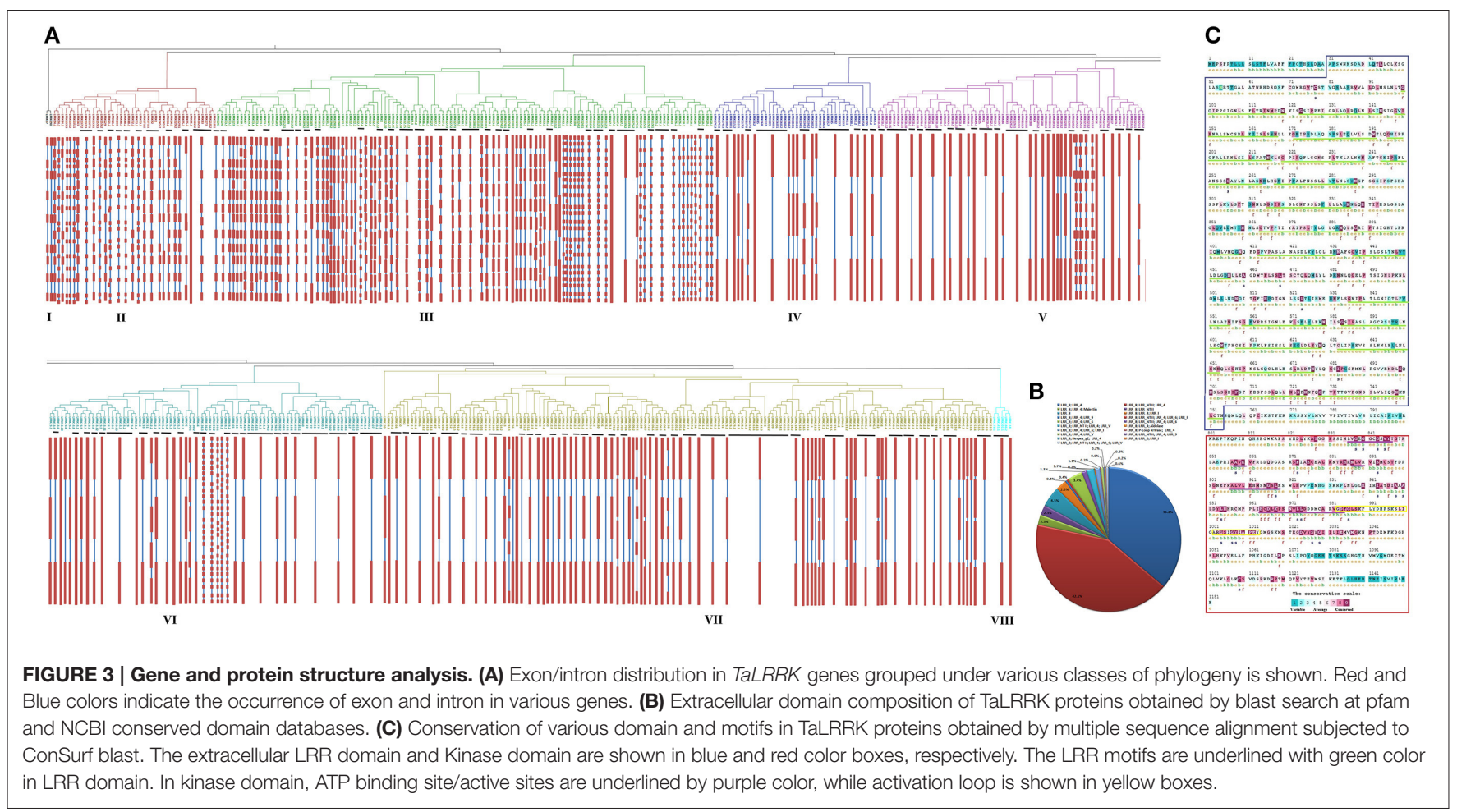

relatively highly expressed in root, spike and leaf, respectively. However, LRRK26 showed similar expression in root and leaf. The phylogenetic grouping of highly expressed genes from various organs indicated that majority of them belong to group III in root (45), stem (30), and spike (26\%). In leaf, high expressing genes were equally $(30 \%)$ distributed in group III and VI, while majority of them (35\%) belongs to group II in grain (Figures 5C-G).

The developmental stage specific expression profiling revealed that the highly abundant genes in root were mostly belonged to $Z \_13$ and $Z \_39$ stages; while in stem, leaf and spike from Z_30, Z_71 and Z_32 stages, respectively. In grain, LRRK79 was exclusively highly expressed in $Z \_71$ and $Z \_75$ stages, while LRRK68 and LRRK90 were highly expressed in $Z$ _85 (Figure 5B). Other stage specific highly expressed genes were LRRK94.3 (root and stem), LRRK180 (Z_23 and Z_71 stage of leaf) and LRRK193.1 (Z_32 stage of spike). The detail expression value of each gene in developmental stage specific manner is listed in File S10.

\section{Gene Expression Analysis under Biotic Stress}

A total of 450 TaLRRK genes were found as differentially expressed ( $\geq 2$ folds change) after infection of fungal pathogens, Blumeria graminis f. sp. tritici (Bgt) and Puccinia striiformis f. sp. tritici (Pst), which causes powdery mildew and stripe rust diseases, respectively (File S11). The top 50 differentially expressed genes were used for further analysis. It was observed that most of the affected genes were up-regulated in Bgt infection, while down-regulated in Pst infection (Figure 6A). A few highly affected genes were- LRRK158, 165.1, and 165.2 (8 fold up), and LRRK202.1 (225 fold down), 134.1-3 (166 fold down) in Pst infection; LRRK148.1 (287 fold up) and 159.2 (183 fold up), and LRRK204 (179 fold down) and 219.4 (140 fold down) in Bgt infection. Further, we observed that $\sim 50 \%$ of highly upregulated genes were exceptionally low expressing during normal uninfected conditions. For instance, the LRRK158, 160.1, and 160.2 showed extremely low expression during normal condition, while these genes were more than 100 fold up-regulated after fungal infection, which indicated their specific role in these stress management.

\section{Gene Expression Analysis under Abiotic Stress}

We studied the expression of TaLRRK genes under heat (HS), drought (DS), and their combined stress (HD) conditions. A total of 492 genes showed $\geq 2$ folds (F) up or down regulation during HS, DS, and HD (File S12), top 50 differentially expressed genes from them were analyzed in detail. Majority of the affected genes $(\sim 50 \%)$ were up-regulated in DS after $1 \mathrm{~h}$, which became either normalized or down-regulated after DS $6 \mathrm{~h}$ (Figure 6B). However, in case of HS, most of the down-regulated genes such as $L R R K 8,61,134$, and 157 , and slightly up-regulated genes like $L R R K 48,166$, and 196 were highly up-regulated in HS $6 \mathrm{~h}$. In case of HD, assorted expression of LRRK genes was observed as compared to the DS and HS. The highly up ( $\uparrow$ ) and down $(\downarrow)$ regulated genes in various stress were- LRRK168 (21 F $\uparrow)$ and LRRK36 ( $2 \mathrm{~F} \downarrow)$ in DS after $1 \mathrm{~h}$, LRRK135.3 (18 $\mathrm{F} \uparrow)$ and LRRK36 (85 F $\downarrow)$ in DS after 6h, LRRK113.1 (30 $\mathrm{F} \uparrow)$ and LRRK $141.2(88 \mathrm{~F} \downarrow)$ in HS after $1 \mathrm{~h}$, LRRK194.2 (44 


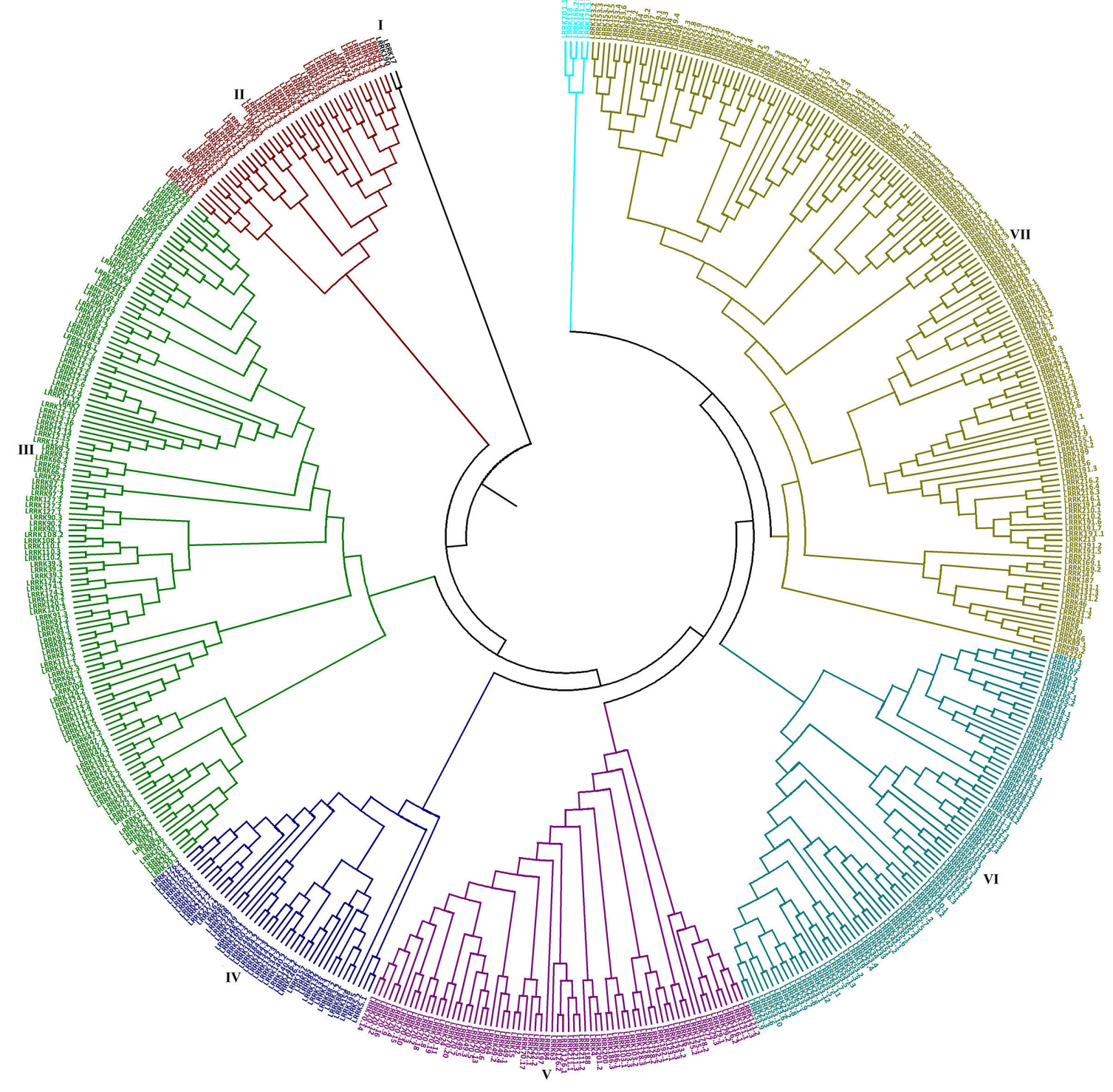

FIGURE 4 | Phylogenetic analysis of TaLRRKs. The evolutionary relationship between TaLRRK proteins was analyzed by neighbor joining method using MEGA 7.0. Figure shows classification of TaLRRK proteins into eight major groups, which are shown with different colors.

$\mathrm{F} \uparrow)$, and LRRK141.3 (137 F $\downarrow)$ in HS after $6 \mathrm{~h}$, LRRK113.1 (15 $\mathrm{F} \uparrow)$ and LRRK180 (146 F $\downarrow)$ in HD after $1 \mathrm{~h}$, and LRRK194.2 (11 F $\uparrow)$ and LRRK141.3 (94 F $\downarrow$ ) in HD after $6 \mathrm{~h}$, respectively. Further, about half of the highly up-regulated genes showed a very low expression during normal condition. To name a few genes such as LRRK168, 113.1, and 135.3 that were extremely low expressing in normal stages, were highly up-regulated in various stresses. The quantitative RT PCR also showed similar expression profile of selected genes (Figure 6F). The LRRK genes 113.2, 180, and 55.3 were down-regulated during each stress, while 26 and 177 were up-regulated in DS_1 h. The phylogenetic grouping showed that majority of these highly affected genes belongs to group VII, followed by IV and VI (Figure 6D).
Salt stress is another major abiotic stress affecting productivity of many crops. Herein, we analyzed the expression pattern of TaLRRK genes in root affected by salt stress using the earlier reported high-throughput RNA sequence data (Zhang et al., 2016). A total of 429 genes were affected by $\geq 2$ folds up or down regulation (File S13), out of which top 50 were considered for detailed analysis. The heat map analysis showed that the highly affected TaLRRK genes could interestingly classified into two contrasting groups of continuously up and down regulated genes over an increase in exposure time of salt (Figure 6C). The RT PCR of selected genes also showed similar expression pattern (Figure 6G). The $L R R K$ genes 26, 31.2, 55.3, 178, and 180 were incrementally up-regulated with exposure time, while LRRK177 was down-regulated. The $L R R K 7,67$, and 135.3 were down 


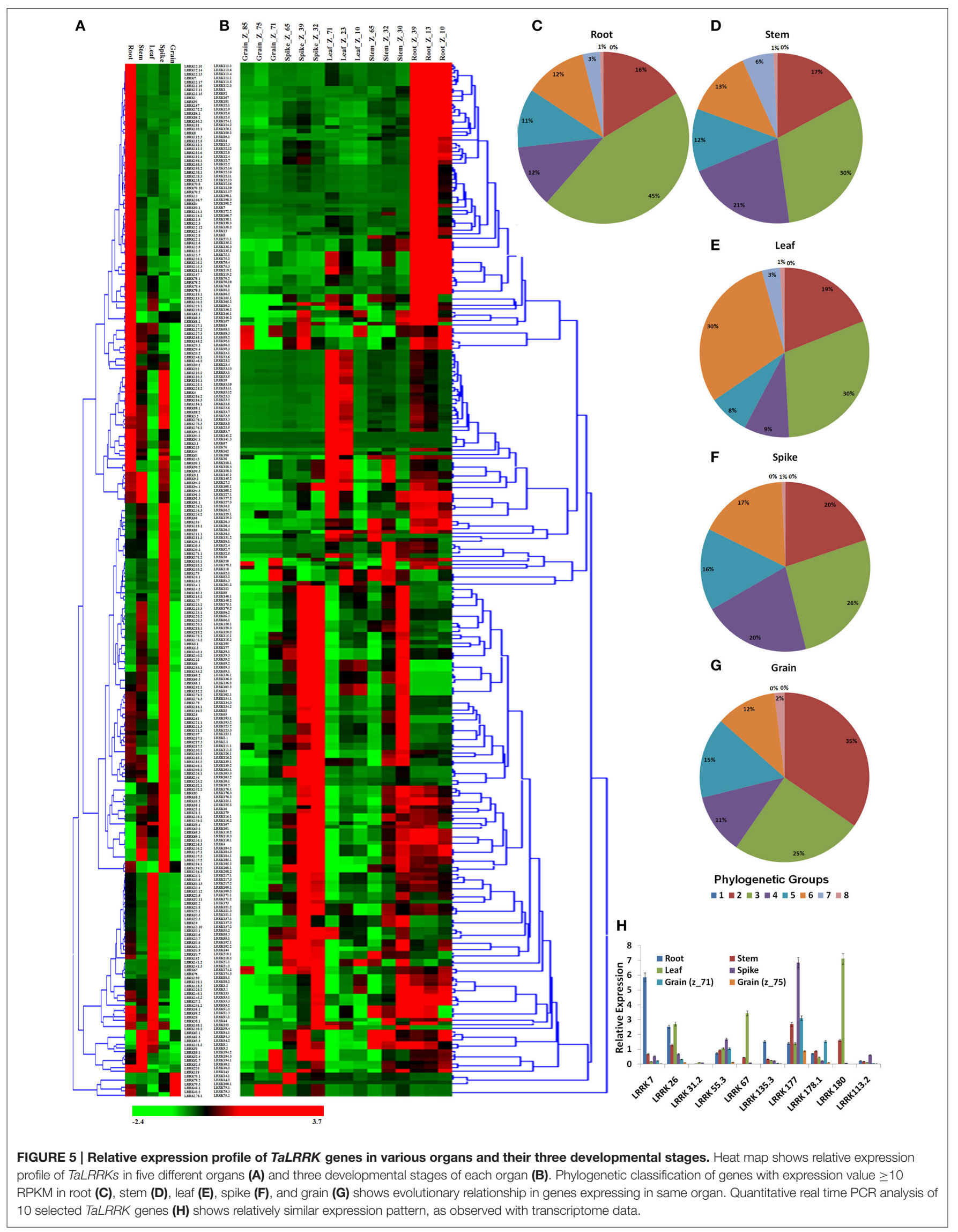




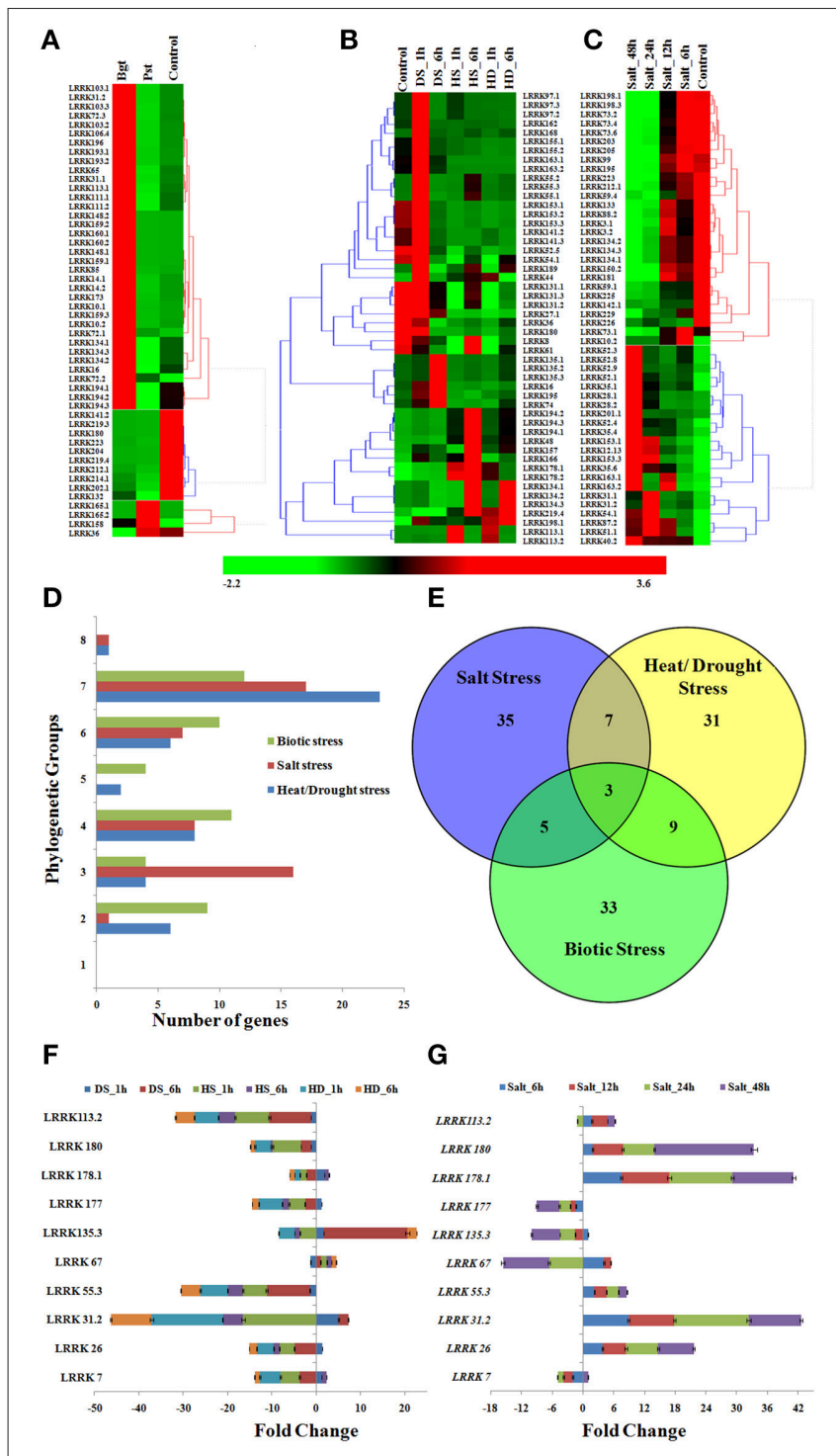

FIGURE 6 | Expression profile of TaLRRK genes under biotic and abiotic stresses. The heat map shows relative expression of top 50 affected genes under (A) biotic stress, (B) heat/drought stress, and (C) salt stress. The symbols are as follows- Bgt; after Blumeria graminis infection, Pst; after Puccinia striiformis inoculation, HS; heat stress, DS; drought stress, HD; combination of heat and drought stress. (D) The phylogenetic classification of top affected genes under biotic and abiotic stresses. (E) The venn diagram shows the number of exclusive and common top affected genes under various biotic and abiotic stresses. Quantitative real time PCR analysis of 10 selected genes under heat/drought (F) and salt (G) stress shows similar expression pattern as detected from transcriptome data analysis.

regulated in most of the treatments, while up-regulated in a few. However, LRRK113.2 was up-reglutated in each treatment except salt_24 h. The phylogenetic classification indicated that most of these up and down regulated genes belonged to group VII and group III, respectively (Figure 6D). A few noticeably up regulated genes were $L R R K 52,55,28$, and 153, while down regulated genes were $L R R K 198,73,205$, and 133. Intriguingly, we observed that some of these highly up-regulated genes (LRRK153.1, 51.1, 31.2) were extremely low expressing under normal conditions, which indicated their specific role in salt stress response.

\section{DISCUSSION}

The LRRKs comprise a major subfamily of RLK. The LRRK genes play vital role in majority of biological activities of plants including growth and developments to stress management (Shiu and Bleecker, 2001; Shiu et al., 2004; Diévart and Clark, 2003). However, their functional characterization has still not been performed in T. aestivum, the most important crop plant of the world. This could be due to several factors like complex allohexaploid $(2 \mathrm{n}=6 \mathrm{x}=42$; AABBDD) genome, unavailability of genome sequence data and others. The availability of chromosome based genome information, and various developmental stage related and stress responsive RNA seq data in recent years facilitated the genome wide characterization of various gene families in T. aestivum (IWGSC, 2014; Zhang et al., 2014, 2016; Liu et al., 2015; Pingault et al., 2015; Shumayla et al., 2016). Since the LRRKs are responsible for various essential functions in plants (Shiu et al., 2004), here we have carried out a comprehensive analysis of these genes in $T$. aestivum.

Blast search of known protein sequences from model plants like arabidopsis, HMM blast and pfam blast are the standard procedures for the identification of new gene family sequences from non-model plants. Similar procedure was followed to characterize $L R R K$ genes from various plants earlier (Zan et al., 2013; Rameneni et al., 2015; Wei et al., 2015). Here, we identified 531 non-redundant TaLRRK genes following the similar procedure, which were further confirmed by blast search against NCBI-conserved domain, PROSITE-Scan and SMART databases. Since the LRRK is the largest subfamily of RLK (Fischer et al., 2016), the number of TaLRRK genes identified was consistent with our expectation. About 213, 234, 303, 309, 382 , and 467 LRRK genes are earlier reported in arabidopsis, tomato, rice, brassica, poplar, and soybean genomes, respectively (Shiu and Bleecker, 2001; Shiu et al., 2004; Sun and Wang, 2011; Zan et al., 2013; Rameneni et al., 2015; Wei et al., 2015; Zhou et al., 2016). The number of TaLRRK genes was fairly higher than the earlier studied plants, which might be due to the large hexaploid genome of T. aestivum (IWGSC, 2014). Similarly the higher number (467) of $L R R K$ genes are reported from soybean, another polyploid crop plant (Schmutz et al., 2010; Zhou et al., 2016).

The allohexaploid genome of $T$. aestivum was originated as a result of hybridization of three (A, B, and D) diploid sub-genomes (Marcussen et al., 2014), which indicates the contribution of each sub-genome in composition of various gene families of $T$. aestivum (Shumayla et al., 2016; Zeng et al., 2016). Further, the contribution of number of genes from a sub-genome would be similar to their number in progenitor genome, unless they are not duplicated or deleted in due course of evolution after polyploidization. The genome wide distribution analysis indicated that the TaLRRK genes were also derived from individual sub-genomes (Figure 1A), 
while their number on $\mathrm{A}$ and $\mathrm{D}$ sub-genomes was quite lower than the number of LRRK genes predicted in T. urartu and Aegilops tauschii, which are progenitors of these sub-genomes, respectively (Marcussen et al., 2014). This indicated deletion of certain TaLRRK genes from these sub-genomes during evolution of T. aestivum after hybridization. The TaLRRK genes were also found to be distributed on each chromosome but at diverse frequency, as earlier reported in various other plants (Rameneni et al., 2015; Wei et al., 2015). For example, maximum and minimum number of TaLRRK genes was located on chromosome 2B and chromosome 5A, respectively. Similarly, chromosome 5 and chromosome 4 in arabidopsis, and chromosome 1 and chromosome 10 in brassica contain the highest and lowest number of $L R R K$ genes, respectively (Rameneni et al., 2015; Wu et al., 2016).

The probability of occurrence of homologous genes has increased thrice in T. aestivum due to its allohexaploid genome (AABBDD). Hypothetically, each T. aestivum gene should have a minimum of three homologous genes, at least one from each subgenome. These may also called as homeologous genes because of their localization on homeologous chromosomes. Here, we observed 531 TaLRRK genes in T. aestivum, which could be grouped into 233 distinct clusters of one or more homologous genes. The total number of TaLRRK genes was not actually thrice to their number in progenitor genomes like T. urartu and Ae. tauschii. Further, we could not detect three homeologous genes in case of each cluster. The majority of TaLRRK genes were single without any homeologous/homologous sequences, while a few had large number (up to 20) of homologous genes. This might be due to the consequences of deletion and/or duplication of certain TaLRRK genes during the process of evolution and acclimatization of T. aestivum. Similar kinds of deletion and duplication events are also reported in other gene families $T$. aestivum (Shumayla et al., 2016; Zeng et al., 2016). Moreover, the number of distinct TaLRRK genes clusters was comparable to the number of $L R R K$ genes reported $\backslash$ predicted in various other diploid plants like arabidopsis, rice, T. urartu, Ae. tauschii and others (Shiu et al., 2004; Sun and Wang, 2011; Rameneni et al., 2015; Wei et al., 2015).

Genome hybridization, duplications, and inter-chromosomal rearrangements of various genes are reported as major events during the evolution of T. aestivum genome (Choulet et al., 2010; Marcussen et al., 2014; Akpinar et al., 2015; Glover et al., 2015). The role of paralogous genes evolved by duplication events (DE) are also reported in the evolution of lectin receptor kinases of T. aestivum (Shumayla et al., 2016), which indicated the role of duplication in the evolvement of other sub-families of RLK in this plant. Therefore, the frequency of various DEs was also analyzed in case of TaLRRK genes, following the established method (Shumayla et al., 2016). A large number of DEs were predicted in case of TaLRRK genes, which were distributed on various subgenomes and chromosomes. About $25 \%$ of DEs were classified as TD and SD, while the majority of them could not further categorized due to the localization of one of both paralogous gene(s) on scaffolds. This is probably due to the unavailability of complete genomic data of T. aestivum. The occurrence of repeat sequences and transposable elements in the genome positively favors the gene duplication during evolution (Magadum et al., 2013). The T. aestivum genome is highly rich in these elements (Choulet et al., 2010, 2014; Marcussen et al., 2014; Glover et al., 2015), which might encourage the DEs in various gene families including TaLRRKs. The role of DEs in the expansion of LRRK gene family is earlier reported in various other plants like poplar, tomato, brassica and arabidopsis (Shiu and Bleecker, 2001; Shiu et al., 2004; Sun and Wang, 2011; Zan et al., 2013; Rameneni et al., 2015; Wei et al., 2015). These results along with earlier reports from other plants indicated that the gene duplication played critical role in diversification and neofunctionalization of LRRK genes during evolution.

The orthologous genes of TaLRRKs in rice and arabidopsis were identified by bidirectional best hit approach. Since both monocots and eudicots are evolved from common ancestor (McClung, 2010), the evolutionary relationship in the form of orthologous genes is obvious between them. Such kind of relationship has been reported in various gene families and plants in earlier studies (Sun and Wang, 2011; Rameneni et al., 2015; Wei et al., 2015; Shumayla et al., 2016).

The functional annotation of TaLRRK genes by blast search against various databases like NCBI-nr, UNIPROT and UNIREF further validated their identity. The TaLRRK sequences showed homology with most of the functionally characterized LRRKs like BRI1, ERL, SERK, CLAVATA from various plants including model plant arabidopsis. These LRRK proteins are earlier reported to be involved in various developmental and stress related activities (Wu et al., 2016). The GO mapping provides information about the characteristic features of various genes across the taxa. Since the LRRKs are membrane proteins (Torii, 2004), the GO analysis indicated membrane bound nature of each TaLRRK protein. Further, the prediction of binding and catalytic properties in TaLRRK proteins supported ligand interacting and enzymatic nature of LRR and kinase domains, respectively (Diévart and Clark, 2004; Torii, 2004). The GO mapping also predicted TaLRRKs role in signaling, stimulus response, and various other metabolic processes, which depicted their diverse function in plant development and defense mechanism. Similar $\mathrm{GO}$ categorization is also reported for brassica LRRKs (Rameneni et al., 2015).

The gene structure analysis showed variation in the presence of number of introns in various TaLRRK genes. The number of introns in TaLRRK genes was varied from zero to a maximum of 27 introns, wherein the majority of genes were single intronic. The variation in the number of exon/intron composition in $L R R K$ genes is reported earlier in various plants. The single intronic $L R R K$ genes are also reported as abundant class in rice, tomato, and poplar. Further, a maximum of 26 introns were reported in poplar, rice and tomato, while 24 in brassica (Sun and Wang, 2011; Zan et al., 2013; Rameneni et al., 2015; Wei et al., 2015). Similar complexity in ERECTA gene structure was reported in arabidopsis (Karve et al., 2011), which was predicted as an ortholog of LRRK69.1 gene of T. aestivum. Previous studies suggested that introns influence the expression of genes, which has been experimentally validated with ERECTA gene in arabidopsis (Morello and Breviario, 2008; Karve et al., 2011; Chorev and Carmel, 2012). The significant expression of a few 
high intronic TaLRRK genes was also observed in certain tissues; however we could not establish a correlation between number of introns and expression pattern.

The length, molecular weight, and pI of TaLRRK proteins also varied as reported in various other plants (Sun and Wang, 2011; Zan et al., 2013; Rameneni et al., 2015; Wei et al., 2015). Since the majority of RLK are membrane protein and associated with the plasma membrane (Torii, 2004). Therefore, presence of a transmembrane region and signal peptide is the signature of the membrane RLK (Shiu and Bleecker, 2001). Occurrence of one or more transmembrane regions and a signal peptide indicated membrane bound nature of most of the TaLRRK proteins and majority of them were localized on plasma membrane. This indicated similar nature of TaLRRK proteins as reported in other plant species (Zan et al., 2013; Rameneni et al., 2015; Wei et al., 2015).

Analysis of domain composition showed the occurrence of 12 different types of domains in TaLRRK proteins, which were found in various combinations, where as kinase domain was consistently present at the C-terminus of each protein (Figure 3B). These diverse domain compositions also indicated functional divergence in various TaLRRK proteins. The diversity in domain composition has also been reported in other plant species like rice and brassica (Sun and Wang, 2011; Rameneni et al., 2015). Mostly the variation in domain composition is found at $\mathrm{N}$-terminus and especially in extra-cellular domain of LRRKs, which supported the deviation in types of signal perception by LRRK proteins. This might be a possible reason for their diverse biological role in plant life cycle. Most of the TaLRRK proteins consisted of numerous types of LRR domains. Though the specific function of each type of LRR domain is not known, but largely they are tandemly repeated leucine rich motifs involved in protein-protein interactions (Bella et al., 2008). Malectin and Ploop NTPase domains were also found in a few TaLRRK proteins, which are probably involved in interaction with glycan and nucleic acid moieties (Leipe et al., 2004; Schallus et al., 2008), however their exact role in LRRK proteins is not established. Similarly a few TaLRRKs consisted of Aldolase II and Herpes_gE domains with unknown function. Several conserved motifs were also predicted in both extracellular LRR and intracellular kinase domains (Figure 3C; Table S2). Most of these motifs are also present in various other plant species (Zan et al., 2013; Rameneni et al., 2015; Wei et al., 2015). The organization of conserved amino acids (GxIPxxLxxLxxLxxLxLxxN) in LRR motif was also found to be highly conserved in both monocot and dicot plants like rice, tomato, and others (Sun and Wang, 2011; Rameneni et al., 2015; Wei et al., 2015). A few conserved motifs were found near the important sites like ATP binding sites, substrate binding sites, and activation loop of kinase domain, which play critical role during signal transduction (Clouse, 2011).

The phylogenetic relationship was analyzed using full-length TaLRRK protein sequences, because it provided excellent evolutionary inference. Full length sequences were used in case of LRRK phylogenetic analyses of other plant species (Sun and Wang, 2011; Rameneni et al., 2015; Wei et al., 2015). The identified homeologous and/or homologous sequences were closely grouped, which further established high sequence homology and evolutionary relation between them. The predicted paralogous sequences were also clustered together, which indicated their similar origin. Similar domain architecture and LRR motifs organization in most of the clades indicated functional correlation between these proteins, which has been observed during expression analysis and discussed below. Further, comparable organization of exon/intron pattern in various sub-groups and clades indicated a strong evolutionary association between them at gene level also. Similar type of gene structure and evolutionary relationship between LRRKs are also reported in other plant species like tomato, rice brassica and poplar (Sun and Wang, 2011; Zan et al., 2013; Rameneni et al., 2015; Wei et al., 2015).

The expression of a gene in a particular organ can be used as a source of information for identifying its function in that organ. Thus, the expression data of 531 TaLRRK genes was extracted from the available whole transcriptome dataset of $T$. aestivum from five different organs (root, stem, leaf, grain, and spike), each with three developmental stages (Pingault et al., 2015). The genes with at least 10RPKM expression values in one or more developmental stages were used for detailed analysis. It might be possible that these genes played critical roles in development of various organs, while the remaining genes might be involved in other functions like stress management. The organ specific expression indicated divergent role of various TaLRRK genes. Most of the genes were specifically expressed in particular organ like leaves or roots or grains, which indicated their specific role in the development of that organ. The organ specific variation in expression of $L R R K$ genes is also reported in arabidopsis, tomato, brassica, soybean, and poplar (Gou et al., 2010; Zan et al., 2013; Rameneni et al., 2015; Wei et al., 2015; Zhou et al., 2016). An evolutionary functional association between organ specific TaLRRK genes was also established as majority of high expressing genes from a particular organ were clustered into same phylogenetic group.

The developmental stage specific expression profiling in various organs indicated that the genes, which were earlier categorized as organ specific, were found to be developmental stage specific. Since the functions of various LRRK proteins of arabidopsis have been established ( $\mathrm{Wu}$ et al., 2016), the putative functions of few TaLRRK proteins were predicted based on their sequence homology. The arabidopsis SERK2 reported to be involved in anther development, maturation and brassinosteroid signaling in roots, anthers, and seedlings (Colcombet et al., 2005; Roux et al., 2011; Wu et al., 2016), showed homology with LRRK94.3, which was also highly expressed in root, grain and spike. Similarly, various other proteins of arabidopsis showed sequence homology with TaLRRK proteins such as BAM1-3 (LRRK60, 122, and 177) and CLAVATA (LRRK41) that are involved in shoot and floral growth, and cell differentiation, GSO (LRRK101) and RPK1 (LRRK116.2) in embryo development, ERL1-2 (LRRK69.2) in organ growth and floral development, BRI1 (LRRK90.1 and 90.4) in shoot vascular development, HSL2 (LRRK107) in abscission of flower and FEI2 (LRRK192.1) in cell wall biosynthesis (Clark et al., 1997; Delgado et al., 2004; Shpak et al., 2004; Deyoung et al., 2006; Nodine et al., 2007; Stenvik et al., 2008; Tsuwamoto et al., 2008; Xu et al., 2008). 
The expression pattern of TaLRRK genes was also studied using high-throughput transcriptome data generated after $24 \mathrm{~h}$ of infection of two common fungal pathogens Bgt and Pst, which are responsible for powdery mildew and stripe rust diseases, respectively (Zhang et al., 2014). We observed a differential response of TaLRRK genes for these two pathogens though both of them are fungal species. This indicated that a diverse mechanism of signal perception and transduction is involved in these two pathogen infections. Further, several highly affected genes during fungal infections were actually very low abundant in normal plant developmental stages, which established their specific role in these biotic stress responses. The differential expression patterns of $L R R K$ genes under fungal attack have also been reported in other plants like poplar (Zan et al., 2013). Though none of the earlier studies have reported characterization of LRRK genes during Pst and Bgt infection, several arabidopsis LRRK proteins are reported to be involved in biotic stress management (Wu et al., 2016). LRRK proteins like PSKR1, BAK1, BRI1, SERK, and FLS of arabidopsis that are involved in various defense responses (Gomez and Boller, 2000; Nam and Li, 2002; Roux et al., 2011; Wang et al., 2011; Mosher et al., 2013; Wu et al., 2016), showed homology with LRRK126.1, 90.3, 188, 39.3, and 220 of T. aestivum, respectively. This indicated that these genes might have similar function as their arabidopsis homologs, which needs to be functionally characterized in future studies.

Plants are exposed to various abiotic stresses like heat, drought, salt and others, which drastically affect the yield by distressing various biochemical and physiological processes (Prasad et al., 2011; Pradhan et al., 2012; Izadi et al., 2014; Yang et al., 2014). Since the LRRKs are reported to be involved in abiotic stress responses (Torii, 2004), we analyzed the effect of various abiotic stresses on the expression of TaLRRK genes using high throughput transcriptome data (Liu et al., 2015; Zhang et al., 2016). Since heat and drought generally act in synergistic manner (Liu et al., 2015), the expression of TaLRRK genes was analyzed under heat, drought, and their combined stresses as well. Several TaLRRK genes were found to be differentially expressed, which indicated their role in these stress conditions. Diverse response of TaLRRK genes during DS and HS indicated variation in their modus operandi. However, a similar trend of up or down regulation was observed for most of the affected genes during HS and DS. In case of HD, majority of genes showed assorted expression, which revealed cross talk between signaling pathway during these stresses. Majority of up-regulated TaLRRK genes were very low expression during normal condition, which indicated their role in these stresses. The phylogenetic analysis revealed that most of the highly affected genes were clustered into group VII (Figure 6D). It is noteworthy that the group VII consisted of least expressing genes during normal stages of various organ development (Figures 5C-G). The results indicated that these group VII genes were not only associated in their expression pattern, but also evolutionarily linked with each other. The role of $L R R K$ genes in abiotic stress management is reported in various plants like rice and potato (Diévart and Clark, 2004; Park et al., 2014; Wu et al., 2016). The OsGIRL1 of rice, which showed homology with LRRK29.1 of T. aestivum, was earlier reported to be involved in heat, salt, osmotic, and gamma radiation stresses (Park et al., 2014).
Salt stress adversely affects the productivity of several crops, and it has been earlier reported that the amount of land affected by salinity is increasing (Wang et al., 2003). Salt stress induces various complex biochemical, molecular, cellular, and physiological changes in plants (Wang et al., 2003; Tuteja, 2007; Munns and Tester, 2008). Salt induced changes in transcriptome expression pattern are earlier reported in the root of T. aestivum (Zhang et al., 2016). The TaLRRK genes were also found to be affected by salt stress. Interestingly, the affected TaLRRK genes could classify into two groups with constant up and down regulation with the exposure time of salt. Most of these up and down regulated genes were clustered into phylogenetic group VII and III, respectively. The results indicated evolutionary association between the genes showing similar behavior during salt stress. The role of $L R R K$ genes in salt stress management is also reported in rice and arabidopsis (Ten Hove et al., 2011; Park et al., 2014).

In conclusion, the present study was focused on the analyses of various characteristic features and expression patterns of TaLRRK genes of T. aestivum, a major food crop. Similar to the other plant species, TaLRRK was found to be one of the largest gene subfamily, which was categorized into eight distinct phylogenetic groups. The gene and protein structure, domain architecture, motifs arrangement, and composition were mostly conserved in various phylogenetic sub-groups. Occurrence of numerous paralogous genes indicated the role of duplication events in the expansion of this gene family during the evolution process. The expression profiling indicated the role of TaLRRK genes in development of various organs under normal and different stress conditions, however the specific role of individual gene needs to be validated by functional genomics tools in future studies. The analysis of major responsive genes in various stress conditions indicated specific roles of most of the TaLRRK genes. For instance, 33, 35, and 31 out of top 50 affected TaLRRK genes were specifically related to biotic, salt, and heat/drought stress, respectively (Figure 6E). Moreover, LRRK134.1, 134.2, and 134.3 genes were affected during all stress conditions. These results may provide valuable information to investigate the function of individual TaLRRK genes in plant development and stress responses using modern genome editing and functional genomics tools.

\section{AUTHOR CONTRIBUTIONS}

SU conceived the idea and designed the experiments. S, SS, and RK performed the experiments. S, VM, KS, and SU analyzed the data. S and SU wrote the manuscript. VM and SU finalized the manuscript.

\section{ACKNOWLEDGMENTS}

Authors are grateful to Panjab University, Chandigarh, India and National Agri-Food Biotechnology Institute, Mohali, Punjab, India for research facilities. Authors are also thankful to URGI and NCBI for the availability of RNA sequences data and various other tools. S is grateful to UGC for Junior Research Fellowship. 


\section{SUPPLEMENTARY MATERIAL}

The Supplementary Material for this article can be found online at: http://journal.frontiersin.org/article/10.3389/fpls.2016. 01374

File S1 | Distribution of 531 LRRKs on T.aestivum A, B, and D sub-genomes and chromosomes.

File S2 | Predicted number of LRRKs in T.urartu and A.tauschii.

File S3 | Unigene clustering, nomenclature, chromosomal localization, and characterization of LRR receptor like kinases.

File S4 | Genome and chromosome wide distribution of Duplication events (paralogs) in LRR receptor like kinases.

File S5 | Predicted orthologs of LRR receptor like kinases in Oryzae sativa and Arabidopsis thaliana.

File S6 | Annotation and gene ontology mapping of LRR receptor like kinases using B2G tool at e-value 10-6.

File S7 | Annotation of LRR receptor like kinases using various databases at e-value 10-6.

\section{REFERENCES}

Afzal, A. J., Wood, A. J., and Lightfoot, D. A. (2008). Plant receptor-like serine threonine kinases: roles in signaling and plant defense. Mol. Plant Microbe Interact. 21, 507-517. doi: 10.1094/MPMI-21-5-0507

Akpinar, B. A., Magni, F., Yuce, M., Lucas, S. J., Simková, H., Safar, J., et al. (2015). The physical map of wheat chromosome 5DS revealed gene duplications and small rearrangements. BMC Genomics 16:453. doi: 10.1186/s12864-015-1641-y

Bella, J., Hindle, K. L., McEwan, P. A., and Lovell, S. C. (2008). The leucine-rich repeat structure. Cell Mol. Life Sci. 65, 2307-2333. doi: 10.1007/s00018-0088019-0

Bhati, K. K., Aggarwal, S., Sharma, S., Mantri, S., Singh, S. P., Bhalla, S., et al. (2014). Differential expression of structural genes for the late phase of phytic acid biosynthesis in developing seeds of wheat (Triticum aestivum L.). Plant Sci. 224, 74-85. doi: 10.1016/j.plantsci.2014.04.009

Chorev, M., and Carmel, L. (2012). The function of introns. Front. Genet. 3:55. doi: 10.3389/fgene.2012.00055

Choulet, F., Alberti, A., Theil, S., Glover, N., Barbe, V., Daron, J., et al. (2014). Structural and functional partitioning of bread wheat chromosome 3B. Science 345:1249721. doi: 10.1126/science.1249721

Choulet, F., Wicker, T., Rustenholz, C., Paux, E., Salse, J., Leroy, P., et al. (2010). Megabase level sequencing reveals contrasted organization and evolution patterns of the wheat gene and transposable element spaces. Plant Cell 22, 1686-1701. doi: $10.1105 /$ tpc. 110.074187

Clark, S. E., Williams, R. W., and Meyerowitz, E. M. (1997). The CLAVATA1 gene encodes a putative receptor kinase that controls shoot and floral meristem size in Arabidopsis. Cell 89, 575-585. doi: 10.1016/S0092-8674(00)80239-1

Clouse, S. D. (2011). Brassinosteroid signal transduction: from receptor kinase activation to transcriptional networks regulating plant development. Plant Cell 23, 1219-1230. doi: 10.1105/tpc.111.084475

Colcombet, J., Boisson-Dernier, A., Ros-Palau, R., Vera, C. E., and Schroeder, J. I. (2005). Arabidopsis SOMATIC EMBRYOGENESIS RECEPTOR KINASES1 and 2 are essential for tapetum development and microspore maturation. Plant Cell 17, 3350-3361. doi: 10.1105/tpc.105.036731

Conesa, A., and Gotz, S. (2008). Blast2GO: a comprehensive suite for functional analysis in plant genomics. Int. J. Plant Genomics 2008:619832. doi: $10.1155 / 2008 / 619832$

Delgado, A. C., Yin, Y., Yu, C., Vafeados, D., García, S. M., Cheng, J. C., et al. (2004). BRL1 and BRL3 are novel brassinosteroid receptors that function
File S8 | Analysis of various domain composition of LRR receptor like kinases.

File S9 | Analysis of domain composition and LRR repeat of LRR receptor like kinases in various phylogenetic groups.

File S10 | Expression analysis of LRR receptor like kinases in various organs and at each developmental stage. Only genes with $10 \mathrm{rpkm}$ value are listed.

File S11 | Expression analysis of LRR receptor like kinases under biotic stress. The genes with 2 fold up or down regulation are shown in pink and green color cells respectively.

File S12 | Expression analysis of LRR receptor like kinases under heat or drought stress. The genes with 2 fold up or down regulation are shown in pink and green color cells respectively.

File S13 | Expression analysis of LRR receptor like kinases during salt stress. The genes with 2 fold up or down regulation are shown in pink and green color cells respectively.

Table S1 | List of primers used for quantitative real time PCR.

Table S2 | Conserved motifs predicted in TaLRRK protein sequences and their corresponding motifs in other plants.

Table S3 | Characterization of TaLRRK proteins in various phylogenetic groups.

in vascular differentiation in Arabidopsis. Development 131, 5341-5351. doi: 10.1242/dev.01403

Deyoung, B. J., Bickle, K. L., Schrage, K. J., Muskett, P., Patel, K., and Clark, S. E. (2006). The CLAVATA1-related BAM1, BAM2 and BAM3 receptor kinase-like proteins are required for meristem function in Arabidopsis. Plant J. 45, 1-16. doi: 10.1111/j.1365-313X.2005.02592.x

Dhanda, S. S., Sethi, G. S., and Behl, R. K. (2004). Indices of drought tolerance in wheat genotypes at early stages of plant growth. J Agron. Crop Sci. 190, 6-12. doi: 10.1111/j.1439-037X.2004.00592.x

Diévart, A., and Clark, S. E. (2003). Using mutant alleles to determine the structure and function of leucine-rich repeat receptor-like kinases. Curr. Opin. Plant Biol. 6, 507-516. doi: 10.1016/S1369-5266(03)00089-X

Diévart, A., and Clark, S. E. (2004). LRR-containing receptors regulating plant development and defense. Development 131, 251-261. doi: 10.1242/dev.00998

Edgar, R. C. (2004). MUSCLE: multiple sequence alignment with high accuracy and high throughput. Nucleic Acids Res. 32, 1792-1797. doi: $10.1093 / \mathrm{nar} / \mathrm{gkh} 340$

Finn, R. D., Bateman, A., Clements, J., Coggill, P., Eberhardt, R. Y., Eddy, S. R., et al. (2014). Pfam: the protein families' database. Nucleic Acids Res. 42, D222-D230. doi: $10.1093 / \mathrm{nar} / \mathrm{gkt} 1223$

Fischer, I., Diévart, A., Droc, G., Dufayard, J. F., and Chantret, N. (2016). Evolutionary dynamics of the Leucine-Rich Repeat Receptor-Like Kinase (LRR-RLK) subfamily in Angiosperms. Plant Physiol. 170, 1595-1610. doi: 10.1104/pp.15.01470

Glover, N. M., Daron, J., Pingault, L., Vandepoele, K., Paux, E., Feuillet, C., et al. (2015). Small-scale gene duplications played a major role in the recent evolution of wheat chromosome 3B. Genome Biol. 16, 188. doi: 10.1186/s13059-0150754-6

Godiard, L., Sauviac, L., Torii, K. U., Grenon, O., Mangin, B., Grimsley, N. H., et al. (2003). ERECTA, an LRR receptor-like kinase protein controlling development pleiotropically affects resistance to bacterial wilt. Plant J. 36, 353-365. doi: 10.1046/j.1365-313X.2003.01877.x

Gomez, L. G., and Boller, T. (2000). FLS2: an LRR receptor-like kinase involved in the perception of the bacterial elicitor flagellin in Arabidopsis. Mol. Cell 5, 1003-1011. doi: 10.1016/S1097-2765(00)80265-8

Gou, X., He, K., Yang, H., Yuan, T., Lin, H., Clouse, S. D., et al. (2010). Genomewide cloning and sequence analysis of leucine-rich repeat receptor-like protein kinase genes in Arabidopsis thaliana. BMC Genomics 11:19. doi: 10.1186/14712164-11-19 
Herve, C., Serres, J., Dabos, P., Canut, H., Barre, A., Rouge, P., et al. (1999). Characterization of the Arabidopsis lecRK-a genes: members of a superfamily encoding putative receptors with an extracellular domain homologous to legume lectins. Plant Mol. Biol. 39, 671-682. doi: 10.1023/A:1006136701595

Hua, D., Wang, C., He, J., Liao, H., Duan, Y., Zhu, Z., et al. (2012). A plasma membrane receptor kinase, GHR1, mediates abscisic acid- and hydrogen peroxide regulated stomatal movement in Arabidopsis. Plant Cell 24, 2546-2561. doi: 10.1105/tpc.112.100107

Izadi, H. M., Rabbani, J., Emam, Y., Pessarakli, M., and Tahmasebi, A. (2014). Effects of salinity stress on physiological performance of various wheat and barley cultivars. J. Plant Nutr. 37, 520-531. doi: 10.1080/01904167.2013.8 67980

Jia, J., Zhao, S., Kong, X., Li, Y., Zhao, G., He, W., et al. (2013). Aegilops tauschii draft genome sequence reveals a gene repertoire for wheat adaptation. Nature 496, 91-95. doi: 10.1038/nature 12028

Jones, D. A., and Jones, J. D. G. (1997). The role of leucine-rich repeats in plant defenses. Adv. Bot. Res. 24, 90-167. doi: 10.1016/S0065-2296(08)60072-5

Karve, R., Liu, W., Willet, S. G., Torii, K. U., and Shpak, E. D. (2011). The presence of multiple introns is essential for ERECTA expression in Arabidopsis. RNA 17, 1907-1921. doi: 10.1261/rna.2825811

Kumar, S., Stecher, G., and Tamura, K. (2016). MEGA7: molecular evolutionary genetics analysis version 7.0 for bigger datasets. Mol. Biol. Evol. 33, 1870-1874. doi: 10.1093/molbev/msw054

Leipe, D. D., Koonin, E. V., and Aravind, L. (2004). STAND, a class of Ploop NTPases including animal and plant regulators of programmed cell death: multiple, complex domain architectures, unusual phyletic patterns, and evolution by horizontal gene transfer. J. Mol. Biol. 343, 1-28. doi: 10.1016/j.jmb.2004.08.023

Li, J., and Tax, F. E. (2013). Receptor-like kinases: key regulators of plant development and defense. J. Integr. Plant Biol. 55, 1184-1187. doi: 10.1111/jipb.12129

Ling, H. Q., Zhao, S., Liu, D., Wang, J., Sun, H., Zhang, C., et al. (2013). Draft genome of the wheat A-genome progenitor Triticum urartu. Nature 496, 87-90. doi: 10.1038/nature11997

Liu, Z., Xin, M., Qin, J., Peng, H., Ni, Z., Yao, Y., et al. (2015).Temporal transcriptome profiling reveals expression partitioning of homeologous genes contributing to heat and drought acclimation in wheat (Triticum aestivum L.). BMC Plant Biol. 15:152. doi: 10.1186/s12870-015-0511-8

Livak, K. J., and Schmittgen, T. D. (2001). Analysis of relative gene expression data using real-time quantitative PCR and the 2-[Delta][Delta] CT method. Methods 25, 402-408. doi: 10.1006/meth.2001.1262

Magadum, S., Banerjee, U., Murugan, P., Gangapur, D., and Ravikesavan, R. (2013). Gene duplication as a major force in evolution. J. Genet. 92, 155-161. doi: 10.1007/s12041-013-0212-8

Marchler, A. B., Derbyshire, M. K., Gonzales, N. R., Lu, S., Chitsaz, F., Geer, L. Y., et al. (2015). CDD: NCBI's conserved domain database. Nucleic Acids Res. 43 , D222-D226. doi: 10.1093/nar/gku1221

Marcussen, T., Sandve, S. R., Heier, L., Spannagl, M., Pfeifer, M., Jakobsen, K. S., et al. (2014). Ancient hybridizations among the ancestral genomes of bread wheat. Science 345:1250092. doi: 10.1126/science.1250092

Matsushima, N., and Miyashita, H. (2012). Leucine-Rich Repeat (LRR) domains containing intervening motifs in plants. Biomolecules 2, 288-311. doi: 10.3390/biom 2020288

McClung, C. R. (2010). A modern circadian clock in the common angiosperm ancestor of monocots and eudicots. BMC Biol. 8:55. doi: 10.1186/17417007-8-55

Morello, L., and Breviario, D. (2008). Plant spliceosomal introns: not only cut and paste. Curr. Genomics 9, 227-238. doi: 10.2174/138920208784533629

Mortazavi, A., Williams, B. A., McCue, K., Schaeffer, L., and Wold, B. (2008). Mapping and quantifying mammalian transcriptomes by RNA-Seq. Nat. Methods 5, 621-628. doi: 10.1038/nmeth.1226

Mosher, S., Seybold, H., Rodriguez, P., Stahl, M., Davies, K. A., Dayaratne, S., et al. (2013). The tyrosine-sulfated peptide receptors PSKR1 and PSY1R modify the immunity of Arabidopsis to biotrophic and necrotrophic pathogens in an antagonistic manner. Plant J. 73, 469-482. doi: 10.1111/tpj.12050

Munns, R., and Tester, M. (2008). Mechanisms of salinity tolerance. Annu. Rev. Plant Biol. 59, 651-681. doi: 10.1146/annurev.arplant.59.032607. 092911
Nam, K. H., and Li, J. (2002). BRI1/BAK1, a receptor kinase pair mediating Brassinosteroid signaling. Cell 110, 203-212. doi: 10.1016/S0092-8674(02)00814-0

Nodine, M. D., Yadegari, R., and Tax, F. E. (2007). RPK1 and TOAD2 are two receptor-like kinases redundantly required for Arabidopsis embryonic pattern formation. Dev. Cell 12, 943-956. doi: 10.1016/j.devcel.2007.04.003

Oliveros, J. C., (2007-2015). Venny. An Interactive Tool for Comparing Lists with Venn's Diagrams. Available online at: http://bioinfogp.cnb.csic.es/tools/venny/index.html

Osakabe, Y., Maruyama, K., Seki, M., Satou, M., Shinozaki, K., and YamaguchiShinozaki, K. (2005). Leucine-rich repeat receptor-like kinase1 is a key membrane-bound regulator of abscisic acid early signaling in Arabidopsis. Plant Cell 17, 1105-1119. doi: 10.1105/tpc.104.027474

Park, S. J., Moon, J. C., Park, Y. C., Kim, J. U., Kim, D. S., and Jang, C. S. (2014). Molecular dissection of the response of a rice leucine-rich repeat receptor-like kinase (LRR-RLK) gene to abiotic stresses. J. Plant Physiol. 171, 1645-1653. doi: 10.1016/j.jplph.2014.08.002

Pingault, L., Choulet, F., Alberti, A., Glover, N., Wincker, P., Feuillet, C., et al. (2015). Deep transcriptome sequencing provides new insights into the structural and functional organization of the wheat genome. Genome Biol. 16, 29. doi: 10.1186/s13059-015-0601-9

Pradhan, G. P., Prasad, P. V. V., Fritz, A. K., Kirkham, M. B., and Gill, B. S. (2012). Effects of drought and high temperature stress on synthetic hexaploid wheat. Funct. Plant Biol. 39, 190-198. doi: 10.1071/FP11245

Prasad, P. V. V., Pisipati, S. R., Momcilovic, I., and Ristic, Z. (2011). Independent and combined effects of high temperature and drought stress during grain filling on plant yield and chloroplast EF-Tu expression in Spring Wheat. J. Agron. Crop Sci. 197, 430-441. doi: 10.1111/j.1439-037X.2011.00477.x

Rameneni, J. J., Lee, Y., Dhandapani, V., Yu, X., Choi, S. R., Oh, M., et al. (2015). Genomic and post-translational modification analysis of leucine-richrepeat receptor-like kinases inBrassica rapa. PLoS ONE 10:e0142255. doi: 10.1371/journal.pone.0142255

Roux, M., Schwessinger, B., Albrecht, C., Chinchilla, D., Jones, A., Holton, N., et al. (2011). The Arabidopsis leucine rich repeat receptor-like kinases BAK1/SERK3 and BKK1/SERK4 are required for innate immunity to hemibiotrophic and biotrophic pathogens. Plant Cell 23, 2440-2455. doi: 10.1105/tpc.111.084301

Schallus, T., Jaeckh, C., Feher, K., Palma, A. S., Liu, Y., Simpson, J. C., et al. (2008). Malectin: a novel carbohydrate-binding protein of the endoplasmic reticulum and a candidate player in the early steps of protein N-glycosylation. Mol. Biol. Cell 19, 3404-3414. doi: 10.1091/mbc.E08-04-0354

Schmutz, J., Cannon, S. B., Schlueter, J., Ma, J. X., Mitros, T., Nelson, W., et al. (2010). Genome sequence of the palaeopolyploid soybean. Nature 463, 178-183. doi: 10.1038/nature08670

Shiu, S. H., and Bleecker, A. B. (2001). Plant receptor-like kinase gene family: diversity, function, and signaling. SciSTKE 113:re22. doi: 10.1126/stke.2001.113.re22

Shiu, S. H., Karlowski, W. M., Pan, R., Tzeng, Y. H., Mayer, K. F., and Li, W. H. (2004). Comparative analysis of the receptor-like kinase family in Arabidopsis and rice. Plant Cell 16, 1220-1234. doi: 10.1105/tpc.020834

Shpak, E. D., Berthiaume, C. T., Hill, E. J., and Torii, K. U. (2004). Synergistic interaction of three ERECTA-family receptorlike kinases controls Arabidopsis organ growth and flower development by promoting cell proliferation. Development 131, 1491-1501. doi: 10.1242/dev.01028

Shumayla, Sharma, S., Pandey, A. K., Singh, K., and Upadhyay, S. K. (2016). Molecular characterization and global expression analysis of lectin receptor kinases in bread wheat (Triticum aestivum). PLOS ONE 11:e0153925. doi: 10.1371/journal.pone.0153925

Soltani, A., Gholipoor, A., and Zeinali, E. (2006). Seed reserve utilization and seedling growth of wheat as affected by drought and salinity. Environ. Exp. Bot. 55, 195-200. doi: 10.1016/j.envexpbot.2004.10.012

Stenvik, G. E., Tandstad, N. M., Guo, Y., Shi, C. L., Kristiansen, W., Holmgren, A., et al. (2008). The EPIP peptide of INFLORESCENCE DEFICIENT IN ABSCISSION is sufficient to induce abscission in Arabidopsis through the receptor-like kinases HAESA and HAESA-LIKE2. Plant Cell 20, 1805-1817. doi: 10.1105/tpc.108.059139

Sun, X., and Wang, G. L. (2011). Genome-wide identification, characterization and phylogenetic analysis of the rice LRR-kinases. PLOS ONE 6:e16079. doi: 10.1371/journal.pone.0016079 
Ten Hove, C. A., Bochdanovits, Z., Jansweijer, V. M., Koning, F. G., Berke, L., Sanchez-Perez, G. F., et al. (2011). Probing the roles of LRR RLK genes in Arabidopsis thaliana roots using a custom T-DNA insertion set. Plant Mol. Biol. 76, 69-83. doi: 10.1007/s11103-011-9769-x

The International Wheat Genome Sequencing Consortium IWGSC. (2014). A chromosome-based draft sequence of the hexaploid bread wheat (Triticum aestivum) genome. Science 345:6194. doi: 10.1126/science. 1251788

Torii, K. U. (2004). Leucine rich repeat receptor kinases in plants: structure, function, and signal transduction pathways. Int. Rev. Cytol. 234, 1-46. doi: 10.1016/S0074-7696(04)34001-5

Torii, K. U., Mitsukawa, N., Oosumi, T., Matsuura, Y., Yokoyama, R., Whittier, R. F., et al. (1996). The arabidopsis ERECTA gene encodes a putative receptor protein kinase with extracellular leucine-rich repeats. Plant Cell 8, 735-746. doi: 10.1105/tpc.8.4.735

Tsuwamoto, R., Fukuoka, H., and Takahata, Y. (2008). GASSHO1 and GASSHO2 encoding a putative leucine-rich repeat transmembrane-type receptor kinase are essential for the normal development of the epidermal surface in Arabidopsis embryos. Plant J. 54, 30-42. doi: 10.1111/j.1365313X.2007.03395.x

Tuteja, N. (2007). Mechanisms of high salinity tolerance in plants. Meth. Enzymol. 428, 419-438. doi: 10.1016/S0076-6879(07)28024-3

Upadhyay, S. K., Dixit, S., Sharma, S., Singh, H., Kumar, J., Verma, P. C., et al. (2013). siRNA Machinery in Whitefly (Bemisia tabaci). PLoS ONE 8:e83692. doi: 10.1371/journal.pone.0083692

Upadhyay, S. K., Sharma, S., Singh, H., Dixit, S., Kumar, J., Verma, P. C., et al. (2015). Whitefly genome expression reveals host-symbiont interaction in amino acid biosynthesis. PLOS ONE 10:e0126751. doi: 10.1371/journal.pone.0126751

Upadhyay, S. K., Singh, H., Dixit, S., Mendu, V., and Verma, P. C. (2016). Molecular characterization of vitellogenin and vitellogenin receptor of Bemisia tabaci. PLoS ONE 11:e0155306. doi: 10.1371/journal.pone. 0155306

Walker, J. C., and Zhang, R. (1990). Relationship of a putative receptor protein kinase from maize to the S-locus glycoproteins of brassica. Nature 345, 743-746. doi: $10.1038 / 345743 \mathrm{a} 0$

Walsh, B. (2003). Population-genetic models of the fates of duplicate genes. Genetica 118, 279-294. doi: 10.1023/A:1024194802441

Wang, W., Vinocur, B., and Altman, A. (2003). Plant responses to drought, salinity and extreme temperatures: towards genetic engineering for stress tolerance. Planta 218, 1-14. doi: 10.1007/s00425-003-1105-5

Wang, Z., Meng, P., Zhang, X., Ren, D., and Yang, S. (2011). BON1 interacts with the protein kinases BIR1 and BAK1 in modulation of temperaturedependent plant growth and cell death in Arabidopsis. Plant J. 67, 1081-1093. doi: 10.1111/j.1365-313X.2011.04659.x

Wei, Z., Wang, J., Yang, S., and Song, Y. (2015). Identification and expression analysis of the $L R R-R L K$ gene family in tomato (Solanum lycopersicum) Heinz 1706. Genome 58, 121-134. doi: 10.1139/gen-2015-0035
Wu, Y., Xun, Q., Guo, Y., Zhang, J., Cheng, K., Shi, T., et al. (2016). Genome-wide expression pattern analyses of the Arabidopsis leucine-rich repeat receptor-like kinases. Mol. Plant 9, 289-300. doi: 10.1016/j.molp.2015.12.011

Xu, S. L., Rahman, A., Baskin, T. I., and Kieber, J. J. (2008). Two leucine-rich repeat receptor kinases mediate signalling linking cell wall biosynthesis and ACC synthase in Arabidopsis. Plant Cell 20, 3065-3079. doi: 10.1105/tpc.108. 063354

Yang, S. J., Zhang, L. Z., Xue, X. Y., Zhang, F. Z., and Shi, Y. S. (2014). Arbuscular mycorrhizal fungi increase salt tolerance of apple seedlings. Bot. Stud. 55, 70. doi: 10.1186/s40529-014-0070-6

Yu, C. S., Chen, Y. C., Lu, C. H., and Hwang, J. K. (2006). Prediction of protein subcellular localization. Proteins 64, 643-651. doi: 10.1002/prot.21018

Yu, C. S., Lin, C. J., and Hwang, J. K. (2004). Predicting subcellular localization of proteins for gram-negative bacteria by support vector machines based on n-peptide compositions. Protein Sci. 13, 1402-1406. doi: 10.1110/ps.03479604

Zan, Y., Ji, Y., Zhang, Y., Yang, S., Song, Y., and Wang, J. (2013). Genome-wide identification, characterization and expression analysis of populus leucinerich repeat receptor-like protein kinase genes. BMC Genomics 14:318. doi: 10.1186/1471-2164-14-318

Zeng, L., Deng, R., Guo, Z., Yang, S., and Deng, X. (2016). Genomewide identification and characterization of Glyceraldehyde-3-phosphate dehydrogenase genes family in wheat (Triticum aestivum). BMC Genomics 17:240. doi: 10.1186/s12864-016-2527-3

Zhang, H., Yang, Y., and Wang, C. (2014). Large-scale transcriptome comparison reveals distinct gene activations in wheat responding to stripe rust and powdery mildew. BMC Genomics 15:898. doi: 10.1186/1471-2164-15-898

Zhang, Y., Liu, Z., Khan, A. A., Lin, Q., Han, Y., Mu, P., et al. (2016). Expression partitioning of homeologs and tandem duplications contribute to salt tolerance in wheat (Triticum aestivum L.). Sci. Rep. 6:21476. doi: 10.1038/srep21476

Zhou, F., Guo, Y., and Qiu, L. J. (2016). Genome wide identification and evolutionary analysis of leucine-rich repeat receptor-like protein kinase genes in soybean. BMC Plant Biol. 16:58. doi: 10.1186/s12870-016-0744-1

Zipfel, C., Kunze, G., Chinchilla, D., Caniard, A., Jones, J. D. G., Boller, T., et al. (2006). Perception of the bacterial PAMP EF-Tu by the receptor EFR restricts Agrobacterium-mediated transformation. Cell 125, 749-760. doi: 10.1016/j.cell.2006.03.037

Conflict of Interest Statement: The authors declare that the research was conducted in the absence of any commercial or financial relationships that could be construed as a potential conflict of interest.

Copyright (๑) 2016 Shumayla, Sharma, Kumar, Mendu, Singh and Upadhyay. This is an open-access article distributed under the terms of the Creative Commons Attribution License (CC BY). The use, distribution or reproduction in other forums is permitted, provided the original author(s) or licensor are credited and that the original publication in this journal is cited, in accordance with accepted academic practice. No use, distribution or reproduction is permitted which does not comply with these terms. 